\title{
Smooth muscle glucose metabolism promotes monocyte recruitment and atherosclerosis in a mouse model of metabolic syndrome
}

\author{
Valerie Z. Wall, ${ }^{1}$ Shelley Barnhart, ${ }^{1}$ Jenny E. Kanter, ${ }^{1}$ Farah Kramer, ${ }^{1}$ Masami Shimizu-Albergine, ${ }^{1}$ \\ Neeta Adhikari, ${ }^{2}$ Thomas N. Wight, ${ }^{3}$ Jennifer L. Hall, ${ }^{2,4}$ and Karin E. Bornfeldt ${ }^{1,5}$ \\ 'Department of Medicine, University of Washington Medicine Diabetes Institute, University of Washington School of \\ Medicine, Seattle, Washington, USA. ²illehei Heart Institute, University of Minnesota, Minneapolis, Minnesota, USA. \\ ${ }^{3}$ Benaroya Research Institute, Matrix Biology Program, Seattle, Washington, USA. ${ }^{4}$ American Heart Association Institute \\ for Precision Cardiovascular Medicine, Dallas, Texas USA. ${ }^{5}$ Department of Pathology, University of Washington Medicine \\ Diabetes Institute, University of Washington School of Medicine, Seattle, Washington, USA.
}

\begin{abstract}
Metabolic syndrome contributes to cardiovascular disease partly through systemic risk factors. However, local processes in the artery wall are becoming increasingly recognized to exacerbate atherosclerosis both in mice and humans. We show that arterial smooth muscle cell (SMC) glucose metabolism markedly synergizes with metabolic syndrome in accelerating atherosclerosis progression, using a low-density lipoprotein receptor-deficient mouse model. SMCs in proximity to atherosclerotic lesions express increased levels of the glucose transporter GLUT1. Cytokines, such as TNF- $\alpha$ produced by lesioned arteries, promote GLUT1 expression in SMCs, which in turn increases expression of the chemokine CCL2 through increased glycolysis and the polyol pathway. Furthermore, overexpression of CLUT1 in SMCs, but not in myeloid cells, accelerates development of larger, more advanced lesions in a mouse model of metabolic syndrome, which also exhibits elevated levels of circulating Ly6C ${ }^{\text {hi }}$ monocytes expressing the CCL2 receptor CCR2. Accordingly, monocyte tracing experiments demonstrate that targeted SMC GLUT1 overexpression promotes Ly6 $\mathrm{C}^{\text {hi }}$ monocyte recruitment to lesions. Strikingly, SMC-targeted GLUT1 overexpression fails to accelerate atherosclerosis in mice that do not exhibit the metabolic syndrome phenotype or monocytosis. These results reveal a potentially novel mechanism whereby arterial smooth muscle glucose metabolism synergizes with metabolic syndrome to accelerate monocyte recruitment and atherosclerosis progression.
\end{abstract}

Conflict of interest: The authors have declared that no conflict of interest exists.

Submitted: August 28, 2017

Accepted: May 1, 2018

Published: June 7, 2018

Reference information: JCI Insight. 2018;3(11):e96544 https://doi.org/10.1172/jici. insight.96544.

\section{Introduction}

Cardiovascular disease (CVD) resulting from atherosclerosis is due in large part to systemic factors, such as increased levels of low-density lipoprotein (LDL) cholesterol, hypertension, smoking, metabolic syndrome, and diabetes. LDL cholesterol is a well-known risk factor for CVD, and the clinical benefit of reducing LDL cholesterol by statins has been comprehensively established in both primary and secondary prevention studies (1-5). Whereas it is clear that systemic factors contribute in major ways to CVD risk, the importance of local processes in the artery wall is becoming increasingly recognized in humans. Thus, recent genomic data from large human studies have identified 5 new CVD risk loci-containing genes that encode proteins expressed in smooth muscle cells (SMCs) as well as proteins involved in inflammatory processes in the artery wall (6). It is therefore likely that systemic factors synergize with processes in arterial SMCs to accelerate atherosclerotic lesion progression to advanced plaques.

Inflammatory processes occur in the lesion during initiation and through progression to advanced clinically relevant plaques, and animal models demonstrate that this, in large part, is due to recruitment of blood monocytes, which become lesional macrophages (7-11). There is an interesting relationship between inflammatory activation of cells and cellular glucose metabolism; some cells alter their metabolism from oxidative phosphorylation to an increased reliance on aerobic glycolysis under inflammatory conditions. For example, inflammatory 
activation of macrophages results in increased glycolysis and increased expression of the non-insulin-dependent glucose transporter GLUT1 (12-14). Inflammatory mediators, such as IL-1 $\beta$, have also been shown to increase glucose uptake and glycolysis in SMCs (15). The use of the glucose analog $\left[{ }^{18} \mathrm{~F}\right]$ fluoro-2-deoxy-D-glucose $\left(\left[{ }^{18} \mathrm{~F}\right]\right.$ FDG) for imaging and identifying actively progressing inflamed lesions in humans $(16,17)$ takes advantage of this process of glucose uptake. Studies in nonhuman primates have revealed that, in addition to lesional macrophages, $\left[{ }^{18} \mathrm{~F}\right] \mathrm{FDG}$ is taken up by medial SMCs in lesioned arteries (18), suggesting increased glucose uptake in these cells in the setting of atherosclerosis. In concordance, we have previously shown that GLUT1 expression is increased in the neointima of rabbit and rat carotid arteries subjected to balloon injury (19).

Whether SMC glucose uptake is a consequence of atherosclerosis or contributes in a causative manner to atherogenesis is unknown. We therefore investigated GLUT1 expression in arterial SMCs in lesioned arteries and whether increased expression of GLUT1 in these cells alters atherosclerosis in a mouse model of metabolic syndrome, which is associated with increased CVD risk in humans.

Our results demonstrate that medial SMCs in close proximity to lesions of atherosclerosis exhibit increased GLUT1 expression and reveal a mechanism whereby arterial smooth muscle glucose metabolism promotes monocyte recruitment and atherosclerosis in a mouse model of metabolic syndrome.

\section{Results}

GLUT1 is upregulated in medial SMCs in close proximity to atherosclerotic lesions and in isolated SMCs exposed to cytokines. LDL receptor-deficient ( $\left(\mathrm{dl}^{-/}\right)$mice fed a high-fat diet for 22 weeks develop large advanced atherosclerotic lesions in the brachiocephalic artery (BCA) (Figure 1A). These BCAs exhibit marked changes to the SMC medial layers underneath the lesion (Figure 1, A and B), such as accumulation of glycosaminoglycans, as indicated by the turquoise alcian blue stain, and loss of the differentiation marker SM $\alpha$-actin (20), suggesting that medial SMCs in proximity to the lesion are less differentiated versus a stable contractile phenotype in the unaffected sections of the media. To assess if GLUT1 expression is altered in SMCs associated with the growing lesion, in situ hybridization for Slc2a1 (gene name for GLUT1) mRNA was conducted. The quantity of Scl2a1 transcripts in medial SMCs beneath the lesion was compared with that in medial SMCs on the opposite side of the BCA from the same animal, where no medial changes or presence of lesion could be observed (Figure 1B). Though the detection of Slc2a1 mRNA varied between animals, expression of the Slc2a1 transcript consistently increased in medial SMCs in close proximity to the lesion, as compared with those that appeared healthy (Figure 1C).

To determine if cytokines associated with the lesion environment could directly increase Slc2a1 mRNA in SMCs, isolated mouse aortic SMCs were treated with TNF- $\alpha$ or IL-1 $\beta$ (both at $5 \mathrm{ng} / \mathrm{ml}$ ) for 24 hours in culture. In addition to cytokines, hypoxia is known to induce GLUT1 in different cell types (21-23). Some SMCs were therefore placed in a hypoxic chamber $\left(1 \% \mathrm{O}_{2}\right)$ for 24 hours as a positive control. Both TNF- $\alpha$ and IL-1 $\beta$ led to a significant increase of Slc2a1 mRNA (Figure 1D). Hypoxia increased Slc2a1 mRNA levels by 5.8 -fold \pm 0.4 -fold (mean \pm SEM; $n=6 ; P<0.0001$ ). TNF- $\alpha$ also significantly increased glucose uptake, measured as ${ }^{3} \mathrm{H}$-2-deoxy-glucose (2-DOG) uptake over a 48-hour period (Figure 1E), demonstrating that the increased GLUT1 expression is of functional significance.

GLUT1 overexpression in SMCS results an increase in glycolysis and polyol pathway intermediates. In order to investigate the functional significance of increased SMC GLUT1 expression, we took advantage of transgenic mice with human SLC2A1 expressed under control of the SMC-targeting SM22 $\alpha$ promoter (24). We crossed these mice with $L d l r^{\prime-}$ mice (generating $L d l r^{-1}$ mice with smooth muscle-specific SLC2A1 expression, herein referred to as SM-GLUT1 mice and WT littermate controls, both deficient in LDLR) to study whether increased glucose flux in SMCs is a causal factor in the process of atherosclerosis in a model of metabolic syndrome. Experimental mice were fed chow diet or a diabetogenic diet with added cholesterol (DDC), which contains saturated fat, sucrose, and $0.15 \%$ added cholesterol $(25,26)$.

Aortic SMCs isolated from SM-GLUT1 mice exhibited an approximate 2-fold increase in glucose uptake, measured as 2-DOG uptake, as compared with SMCs from littermate controls (Figure 2A), demonstrating that the GLUT1 transgene was functional. A small but significant increase in lactate release, a result of increased aerobic glycolysis, was also observed in SMCs from the SM-GLUT1 mice, as compared with littermates (Figure $2 \mathrm{~B}$ ). Consistently, we have previously shown, using ${ }^{13} \mathrm{C}$-nuclear magnetic resonance spectroscopy, that GLUT1 overexpression in SMCs results in increases in the contribution of glucose to lactate and acetyl-CoA formation $(19,27)$. To further investigate the effect of GLUT1 overexpression in SMCs, metabolomics studies were conducted in mouse aortic SMCs transduced with a GLUT1 retroviral 

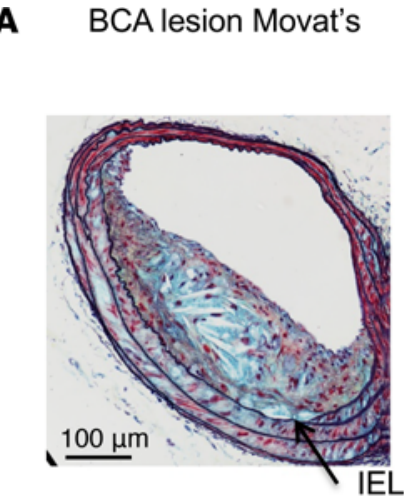

\section{Arterial SMC S/c2a1 mRNA}

D SMC SIc2a1 mRNA
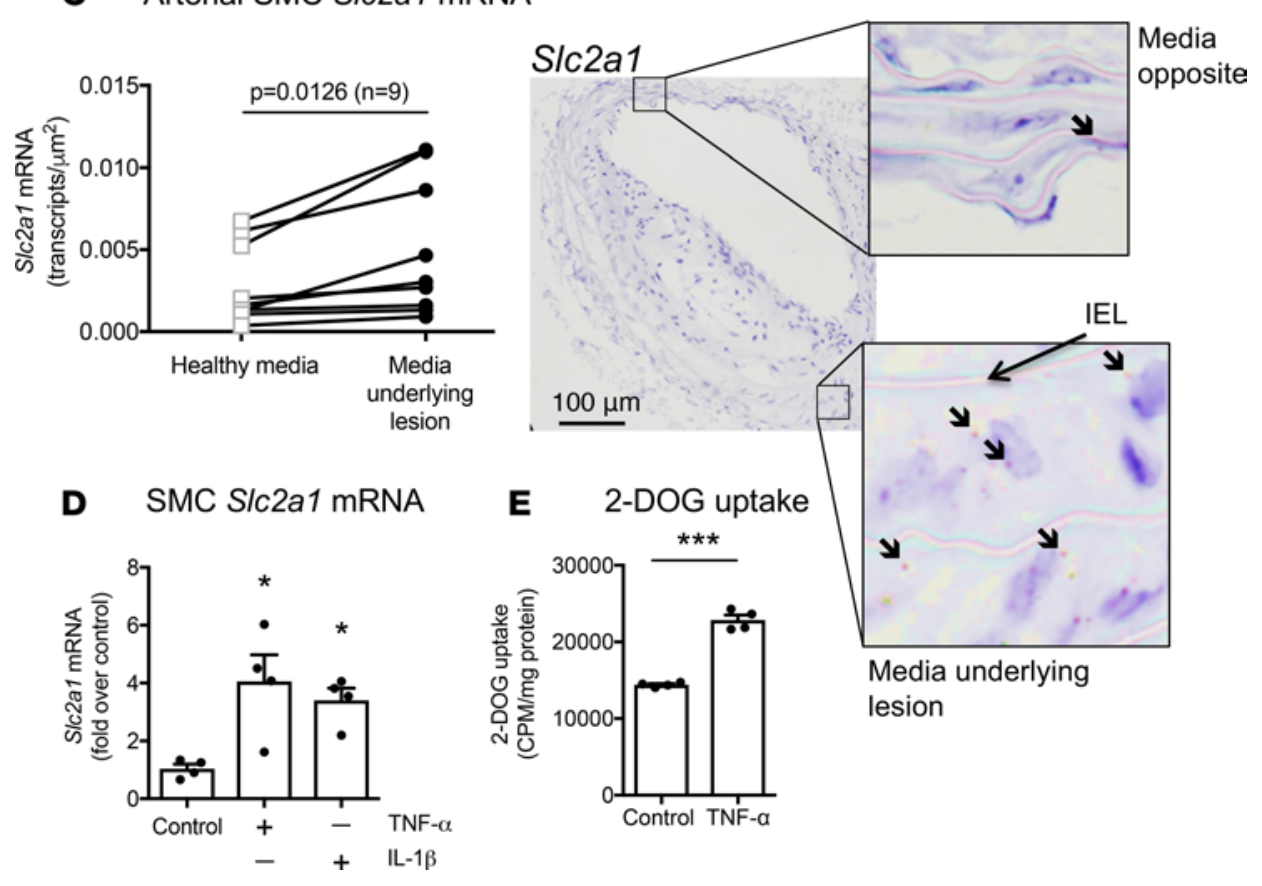

B BCA lesion SM $\alpha$-actin

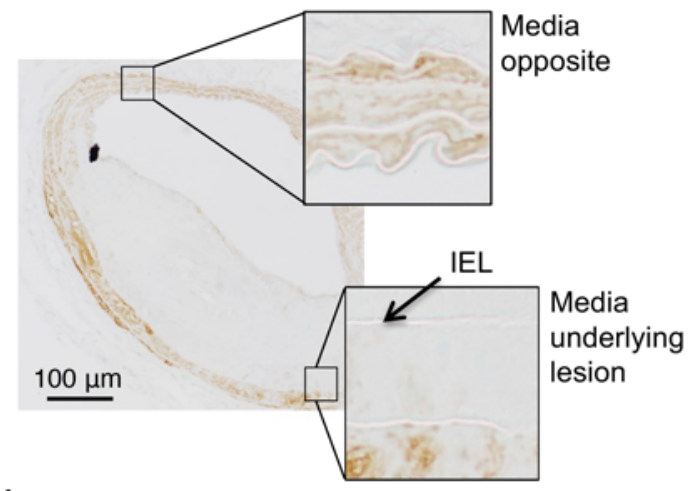

Figure 1. GLUT1 is increased in medial SMCs underlying lesions. Representative advanced BCA lesion stained with a Movat's pentachrome stain (A) and a SM $\alpha$-actin antibody (B) after 22 weeks of DDC feeding. (C) S/c2a1 mRNA transcripts were quantified by in situ RNA hybridization in the medial SMCs beneath the lesion and compared with transcripts from medial SMCs at a nonatherosclerotic site opposite from the lesion in the same animals. SIc2a1-positive signals are indicated by arrows. (D) SIc2a1 mRNA is increased after a 24-hour stimulation with TNF- $\alpha$ (5 $\mathrm{ng} /$ $\mathrm{ml})$ or IL-1 $\beta(5 \mathrm{ng} / \mathrm{ml})$. (E) 2-Deoxyglucose (2-DOG) uptake is increased after 48-hour stimulation with $5 \mathrm{ng} / \mathrm{ml}$ TNF- $\alpha$. Results are expressed as mean \pm SEM ( $n=4$ in D and E). Statistical analysis was performed by 2-tailed paired (C) or unpaired (E) Student's $t$ test or 1-way ANOVA and Tukey's post hoc tests $(\mathbf{D}) ;{ }^{*} P<0.05 ;{ }^{* * *} P<0.001$. IEL, internal elastic lamina.

vector (Figure 2, C-I). Increased Slc2a1 mRNA levels were achieved in SMCs, as compared with the empty pBM vector transduced control SMCs (Figure 2C). Increased glucose flux in the GLUT1 overexpressing SMCs was demonstrated as an increase in lactate release (Figure 2D), similar to the increase in SMCs from the SM-GLUT1 mice (Figure 2B), and was further verified by an increased expression of thioredoxin interacting protein (Txnip) mRNA (Figure 1E), which encodes a protein known to be regulated by glycolytic flux (28) and to be induced by elevated glucose in SMCs (29).

To better understand GLUT1-mediated changes in glucose metabolism in SMCs, we used targeted liquid chromatography-tandem mass spectrometry (LC-MS/MS) to quantify 138 intermediates in glycolysis, polyol pathway, pentose phosphate pathway, TCA cycle, amino acid metabolism, nucleotide metabolism, vitamins, redox regulation, bile acid metabolism, fatty acid metabolism, urea cycle, oxidative damage, and polyamine metabolism. GLUT1 overexpression resulted in a marked increase in cellular glucose levels (Figure 2F), as expected. Interestingly, the only other significant increases observed in GLUT1-overexpressing SMCs, after correction for multiple comparisons, were in levels of sorbitol and glyceraldehyde (Figure 2, G and H), intermediates in the polyol pathway. GLUT1 overexpression did not significantly alter levels of glucosamine (Figure 2I), and markers of oxidative stress were not significantly elevated by GLUT1 overexpression after multiple-comparison correction. The full metabolomics data set is shown in Supplemental Table 1 (supplemental material available online with this article; https://doi.org/10.1172/jci.insight.96544DS1). Figure 2J shows a schematic representation of the metabolomics data and biochemical measurements. 
A 2-DOG uptake

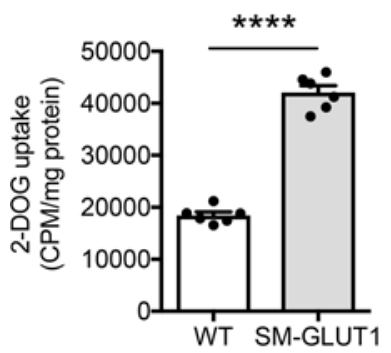

F Glucose

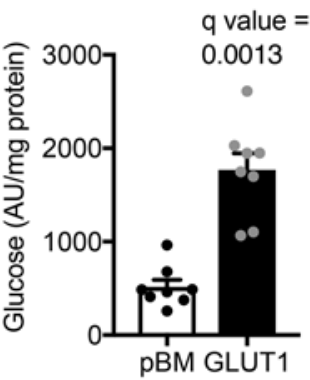

H
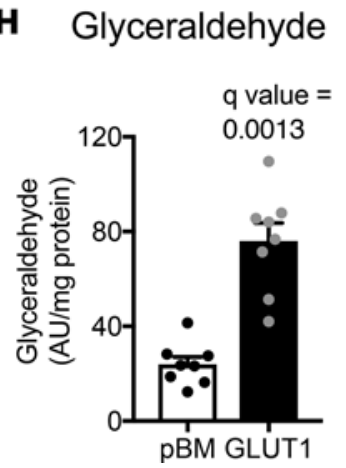

B Lactate release

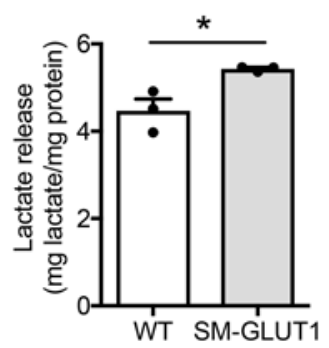

G

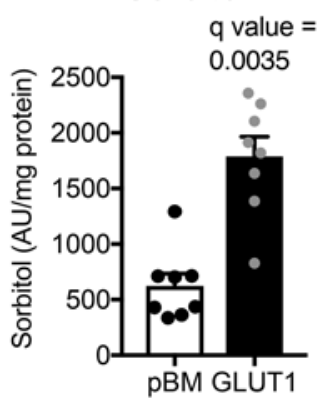

I Glucosamine

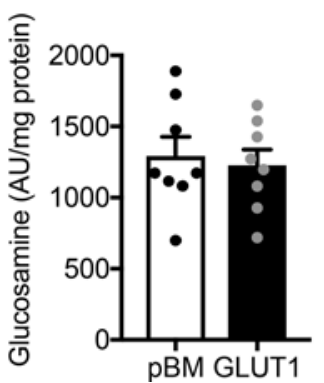

C Slc2a1 mRNA

D Lactate release

E Txnip mRNA
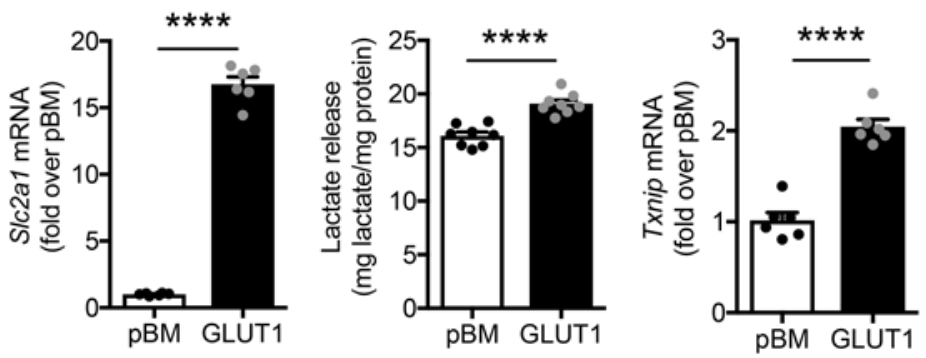

\section{J Schematic representation}

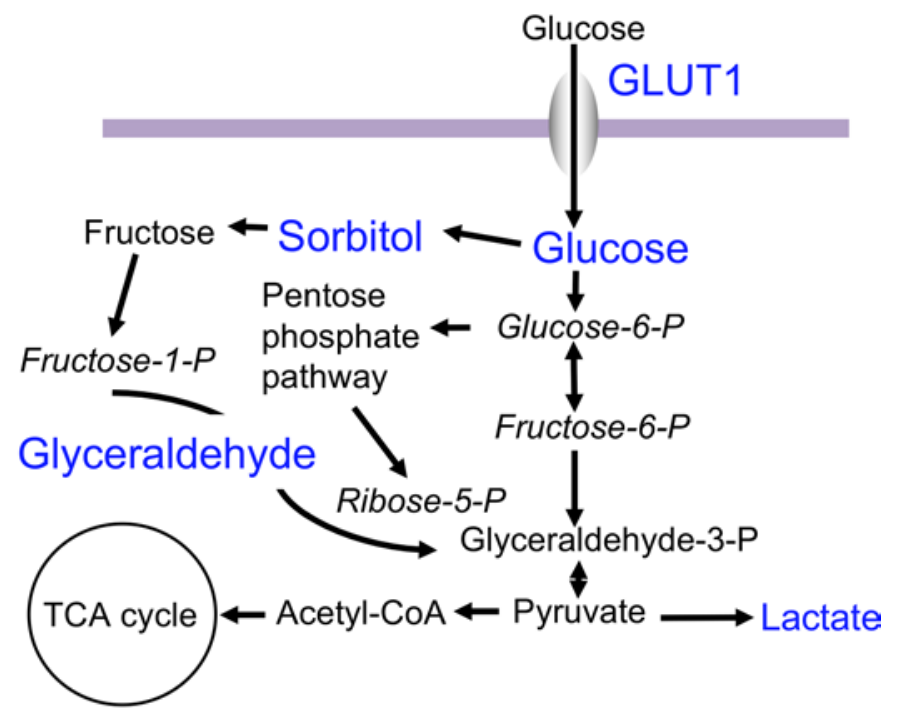

Figure 2. SMC GLUT1 overexpression leads to increased glycolysis and accumulation of polyol pathway intermediates. Aortic SMCs were isolated from LdIr $^{-/-}$SM-GLUT1 mice and littermate controls, and 2-DOC uptake (A) and lactate release (B) were measured. In other experiments, GLUT1 (SIc2a1) was overexpressed by a pBM retrovirus (C), leading to increased lactate release (D) and Txnip mRNA levels (E). Targeted metabolomics revealed that GLUT1 overexpression increased glucose levels (F) and the polyol pathway intermediates sorbitol (G) and glyceraldehyde (H). GLUT1 overexpression did not significantly increase glycosamine pathway intermediates (I). Results are expressed as mean \pm SEM ( $n=6$ in $\mathbf{A} ; n=3$ in $\mathbf{B} ; n=6$ in $\mathbf{C}$ and $\mathbf{E} ; n=8$ in $\mathbf{D}$ and $\mathbf{F}-\mathbf{I})$. Statistical analysis was performed by 2-tailed unpaired Student's $t$ test in A-E and unpaired $t$ tests corrected for multiple comparisons using the Holm-Sidak method (adjusted $q$ values are shown); ${ }^{*} P<0.05 ;{ }^{* * * *} P<0.0001$. (J) Schematic representation of the results of GLUT1 overexpression on SMC metabolism. Significantly increased metabolites are shown in blue, while nominally significantly increased metabolites are highlighted by italics.

Ldlr'- mice fed a high-fat, high-sucrose diet develop a metabolic syndrome phenotype, which is not affected by $S M$-GLUT1 overexpression. Our first atherosclerosis study in WT and SM-GLUT1 Ldl $r^{-1}$ mice was performed in male and female mice fed chow or DDC for 16 weeks (Figure 3A). In male mice, DDC feeding resulted in significantly increased plasma cholesterol (Figure 3B) and plasma triglycerides (Figure 3C), as expected. During 16 weeks of DDC feeding, the animals also gained weight (Figure 3D), had modestly elevated nonfasting blood glucose (Figure 3E), and had developed glucose intolerance by week 13 (Figure $3 \mathrm{~F}$ ). Female mice had a metabolic syndrome phenotype induced by DDC feeding similar to that of male mice. Plasma cholesterol levels were equally high in male and female mice fed DDC (Figure 3G). DDC-fed female mice also exhibited elevated plasma triglycerides (Figure $3 \mathrm{H}$ ), gained weight, and developed slightly elevated nonfasting blood glucose and glucose intolerance, but not to the same degree as did male mice (Figure 3, I-K). Levels of glycated hemoglobin ( $\mathrm{HbA1c}$ ) remained in the normal range in both DDC-fed male and female mice (Figure 3L). Importantly, there was no effect of genotype between SM-GLUT1 and WT mice for any of these systemic measurements in male or female mice. Thus, DDC 
A Study design

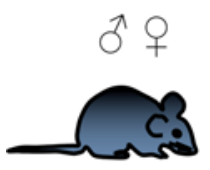

B Cholesterol $\delta$

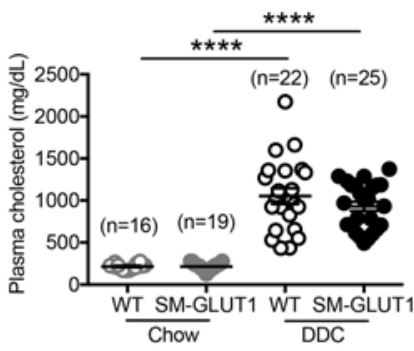

c Triglycerides $\delta$

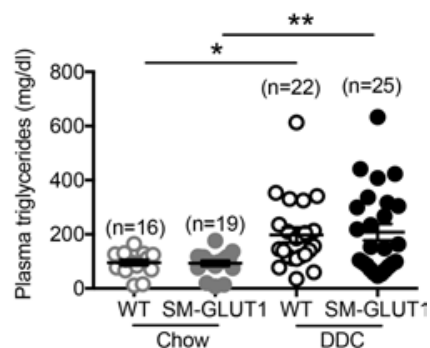

D Body weight $\lesssim$

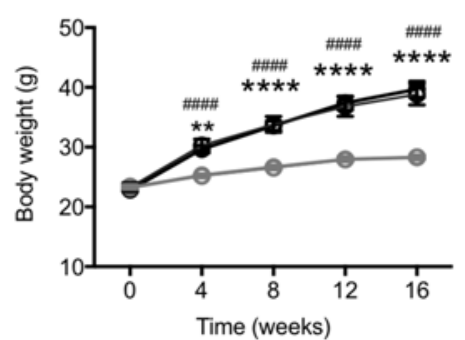

G Cholesterol $q$

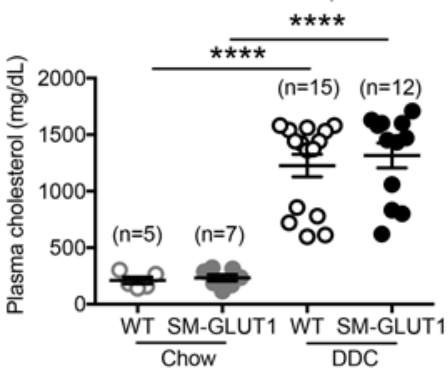

J Blood glucose +

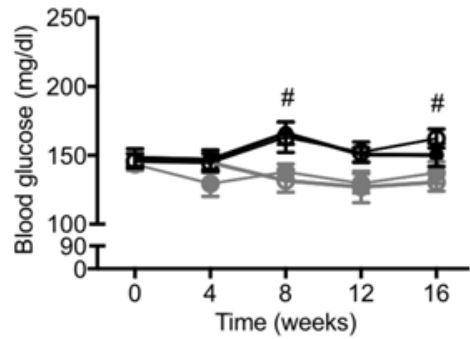

E Blood glucose $\sigma^{\lambda}$

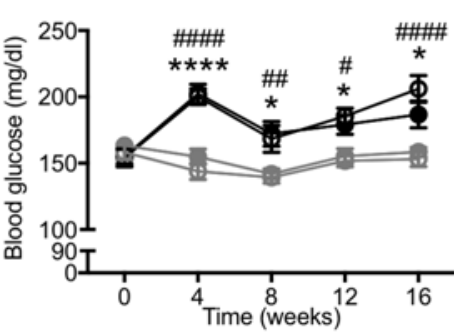

H Triglycerides $q$

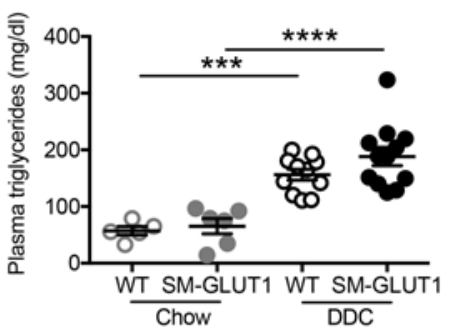

K GTT +

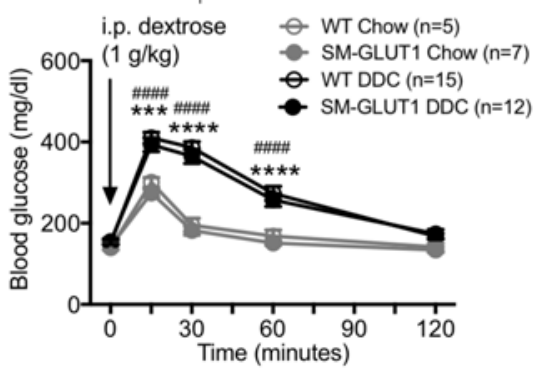

F GTT $\delta$

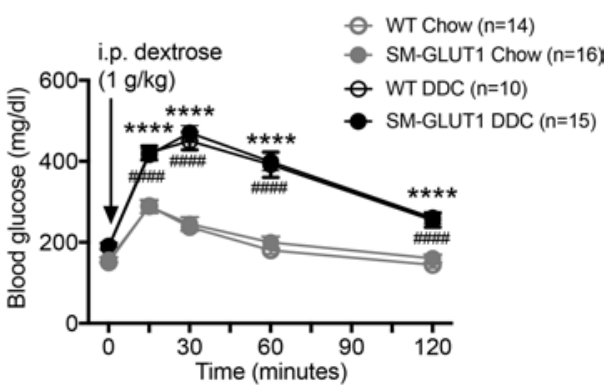

I Body weight ㅇ

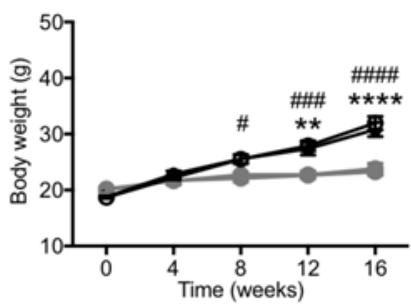

L $\quad H b A 1 c$

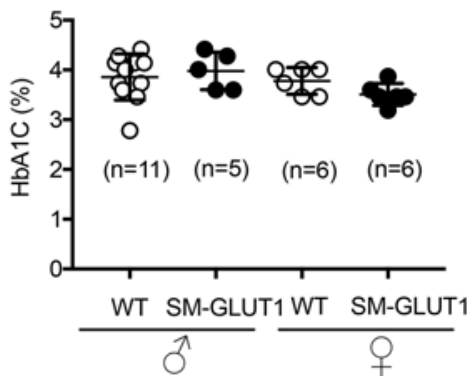

Figure 3. DDC feeding in male and female LdIr/- mice results in dyslipidemia and features of metabolic syndrome, which are not affected by SMC GLUT1 overexpression. (A) Male and female WT and SM-CLUT1 Ldlr ${ }^{-1-}$ mice, 8-10 weeks of age, were fed DDC or chow for 16 weeks. DDC-fed male mice exhibited elevated plasma cholesterol (B), elevated plasma triglycerides (C), increased body weight (D), and elevated nonfasting blood glucose levels (E). (F) Glucose tolerance tests revealed impaired glucose tolerance. SM-GLUT1 overexpression did not affect any of these parameters. Results are expressed as mean \pm SEM ( $n=16-25$ for groups in $\mathbf{D}$ and $\mathbf{E}$, as shown in $\mathbf{B}$ and $\mathbf{C} ; n=10-16$ in $\mathbf{F})$. Female $L d l r^{-/}$mice fed DDC develop similarly elevated plasma cholesterol (C) but exhibit a lesser degree of increased triglycerides (H), body weight gain (I), nonfasting blood glucose (J), and glucose intolerance (K), as compared with male mice. (L) HbA1c levels in male and female mice fed DDC. Results are expressed as mean \pm SEM ( $n=5-15$ in G-K, as shown in G). Statistical analysis was performed by 1-way ANOVA (B, C, G, H) or 2-way ANOVA (D-F, I-K) with Tukey's post hoc test; ${ }^{*}, \#$ P

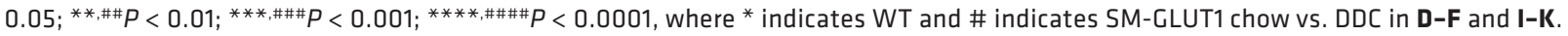


A Aortic lesion

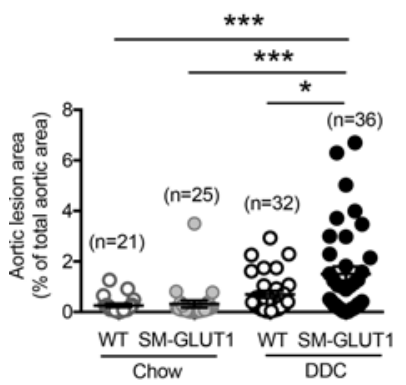

B Aortic cholesterol

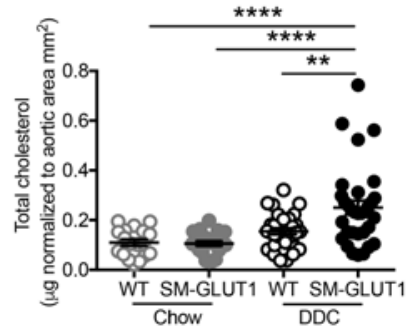

C Aortic free cholesterol

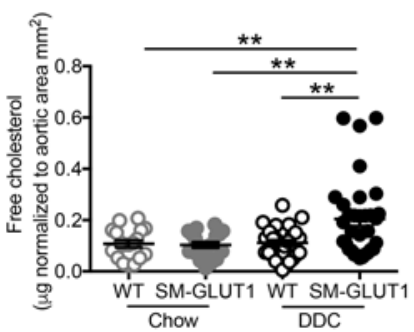

D Aortic CE

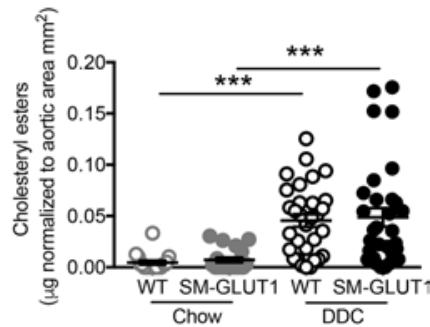

E Aortic Abca1

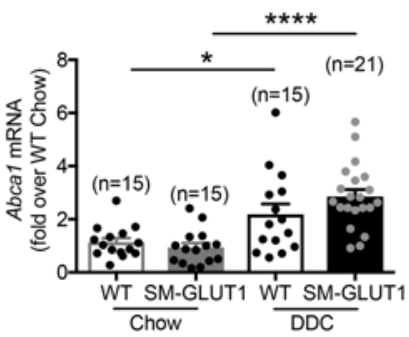

H Aortic Cnn1

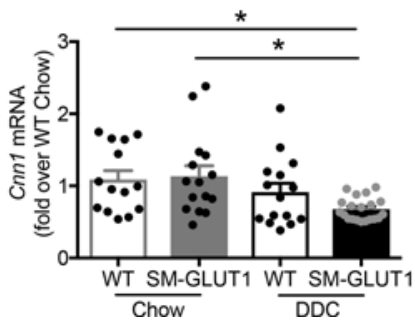

F Aortic $\mathrm{Cxcl1}$

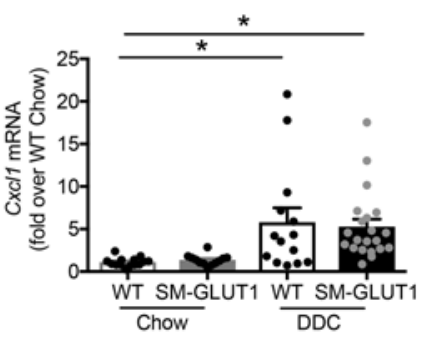

I Aortic Has1

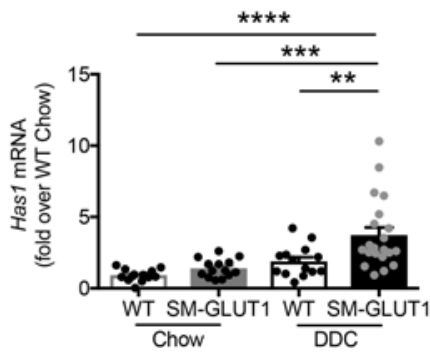

G Aortic Cd11b

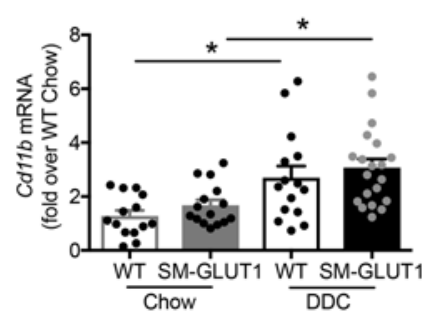

J Aortic Has3

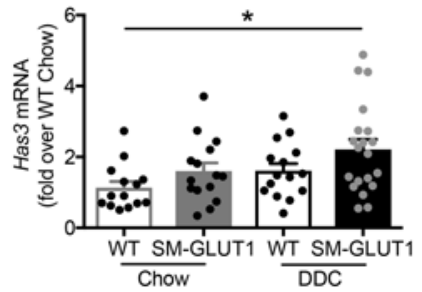

Figure 4. Smooth muscle-targeted GLUT1 overexpression enhances aortic lesion size and complexity in DDC-fed mice. (A) SM-GLUT1 mice fed DDC exhibit larger aortic lesions. Lipids extracted from the whole aorta revealed greater cholesterol content (B) in SM-CLUT1 mice fed DDC. Free cholesterol (C) accounted for the increase, whereas cholesteryl esters (CE) (D) were raised by DDC feeding alone. Gene expression was measured by real-time PCR in homogenized whole aortas: $\operatorname{Abca1}(\mathbf{E}), C x c 11(\mathbf{F}), \operatorname{Cd11b}(\mathbf{C}), \operatorname{Cnn1}(\mathbf{H}), \operatorname{Has1}(\mathbf{I})$, and Has3 (J). Results are expressed as mean \pm SEM $(\mathbf{A}-\mathbf{D}, n=21-36$, as shown in A; E-J, $n=$ 15-21 as shown in E). One mouse in the SM-GLUT1 DDC group was excluded as a statistical outlier based on Grubb's test. Statistical analysis was performed by 1-way ANOVA with Tukey's post hoc test; ${ }^{*} P<0.05 ;{ }^{* *} P<0.01 ;{ }^{* *} P<0.001 ;{ }^{* * *} P<0.0001$.

feeding results in a phenotype similar to that frequently seen in metabolic syndrome, and this phenotype was not affected by smooth muscle GLUT1 overexpression.

Smooth muscle GLUT1 overexpression accelerates atherosclerosis in a mouse model of metabolic syndrome. We next investigated if increased SMC GLUT1 expression could exacerbate atherosclerosis in the aortas of male and female mice fed either chow or DDC for 16 weeks. Neither genotype developed aortic lesions when fed the regular chow diet (Figure 4A), demonstrating that SMC-targeted GLUT1 expression is not sufficient to induce atherosclerosis. DDC feeding of WT mice resulted in small lesions at this 16-week time point, which were not significantly different from either of the chow-fed groups. Interestingly, SM-GLUT1 mice exhibited aortic lesions that were statistically larger than both of the chow-fed groups and those of WT mice fed DDC (Figure 4A). Lipids extracted from aortas support the increased lesion area in the SM-GLUT1 mice fed DDC. Thus, SM-GLUT1 mice fed DDC exhibited increased aortic cholesterol (Figure 4B). Most of the elevated aortic cholesterol in the DDC-fed SM-GLUT1 mice was due to significantly more free cholesterol compared with the WT mice fed DDC and the chow-fed mice (Figure 4C), suggesting more advanced features of lesions $(30,31)$. Both WT mice and SM-GLUT1 mice fed DDC had elevated aortic cholesteryl esters reflective of lipid-loaded foam cells (Figure 4D). 
Following lipid extraction, mRNA was isolated from the entire aorta to analyze gene expression. Gene expression in aortas demonstrates effects of DDC feeding, such as elevated levels of the cholesterol transport protein $A b c a 1$, the chemokine $C x c l 1$, and the myeloid cell marker $C d 11 b$ (Figure 4, E-G), suggesting increased lipid loading and aortic inflammation. These markers were not affected by smooth muscle GLUT1 overexpression. Levels of mouse GLUT1 (Slc2a1) were not affected by the human GLUT1 transgene (Supplemental Figure 1A). Importantly, levels of human SLC2A1 in the transgenic mice were very low, as compared with a human artery sample, suggesting that the GLUT1 transgene is unlikely to lead to artificial effects due to supraphysiological levels of overexpression (Supplemental Figure 1B; see complete unedited blots in the supplemental material). Furthermore, the human GLUT1 transgene did not alter other GLUT isoforms shown to be expressed in SMCs (32), including GLUT9, GLUT10, and GLUT12 (Supplemental Figure 1, C-F).

The possibility of phenotypic switching of SMCs from a contractile to a synthetic phenotype was assessed by measurements of smooth muscle actin (Acta2) and calponin (Cnn1) mRNA. SM-GLUT1 mice fed DDC exhibited a significant reduction in Cnn1, as compared with chow-fed mice (Figure $4 \mathrm{H}$ ), while no overall reduction in Acta2 was detected (data not shown). These results suggest that GLUT1 overexpression does not promote an overall dedifferentiation of the SMCs but might facilitate a change in SMC phenotype when in combination with DDC feeding. Moreover, DDC-fed SM-GLUT1 mice exhibited elevated levels of the hyaluronan synthetases Has1 and Has3, but not Has2 (Figure 4, I and J and data not shown). Increased hyaluronan synthesis could provide an environment permissive for SMC migration and proliferation (33). The increases in Has 1 and Has3 are likely to be due to the inflammatory lesion environment in DDC-fed mice, rather than to a direct effect of SMC GLUT1 overexpression, as GLUT1 overexpression did not increase hyaluronan accumulation in mice after 4 weeks of DDC feeding, before lesions have formed (data not shown).

Morphological features of the lesions were characterized by histological analysis of BCA cross-sections from chow-fed mice and a subset of DDC-fed mice with a pronounced metabolic phenotype. These DDC-fed mice had plasma cholesterol levels $>1,000 \mathrm{mg} / \mathrm{dl}$ and an average weight gain of $43.4 \% \pm 1.4 \%$ (mean \pm SEM; $n=41$ ). DDC-fed mice with plasma cholesterol levels below 1,000 mg/dl had gained less weight $38.2 \% \pm 1.3 \%(P<0.01 ; n=32)$, suggesting a weaker metabolic phenotype. The entire BCA was serial sectioned, and one-sixth of the sections across the entire BCA were assessed for average medial size, lesion size, and the fraction of these sections containing different morphological features. Representative images for each group are shown in Figures 5, A and B, and demonstrate that chow-fed mice exhibit no lesions, regardless of genotype; that DDC-fed WT mice have small fatty streak-type lesions; and that DDC-fed SM-GLUT1 mice have more advanced lesions, some of which demonstrated fibrous caps and necrotic cores. Consistently, SM-GLUT1 mice fed DDC had significantly larger BCA lesions than both of the chow-fed groups and the WT mice fed DDC (Figure 5C), consistent with the aortic lesion data. Neither genotype nor diet altered the average medial area (Figure 5D).

Sections adjacent to the maximal BCA lesion site were evaluated for the presence of macrophages and SMCs by immunohistological staining, using Mac- 2 and SM $\alpha$-actin antibodies, respectively. The lesions were primarily composed of these 2 cell types (Figure 5G). Macrophage and SMC content in BCA lesions were both increased in SM-GLUT1 mice fed DDC, as compared with chow-fed mice and DDC-fed WT mice (Figure 5, E and F).

Next, several morphological features of BCA media and lesions were evaluated. As shown in Figures 5 , $\mathrm{H}-\mathrm{J}$, the frequencies of necrotic cores and intraplaque hemorrhage - both indicators of advanced lesions — were significantly higher in DDC-fed SM-GLUT1 mice. A BCA lesion showing intraplaque hemorrhage from a DDC-fed SM-GLUT1 mouse is shown in Figure 5H. Several other features of advanced lesions were also restricted to DDC-fed SM-GLUT1 mice (Supplemental Table 2).

Overall, these results demonstrate that smooth muscle GLUT1 overexpression accelerates both atherosclerotic lesion growth and severity in the mouse model of metabolic syndrome.

The proatherogenic effect of SM-GLUT1 overexpression is selective to SMCs and requires a metabolic syndrome phenotype. We have previously shown that overexpression of GLUT1 in myeloid cells is not sufficient to drive inflammation or atherosclerosis in lean $\mathrm{Ldll}^{-1-}$ mice fed a low-fat diet (14). In the current study on SMCs in a metabolic syndrome model, the effect of GLUT1 required DDC feeding, making it possible that increased GLUT1 expression in myeloid cells would likewise promote atherosclerosis under these conditions. To investigate the cell type specificity of GLUT1 overexpression on atherosclerosis, we repeated the 16-week DDC-feeding atherosclerosis study in mice that had received 
A BCA WT Chow

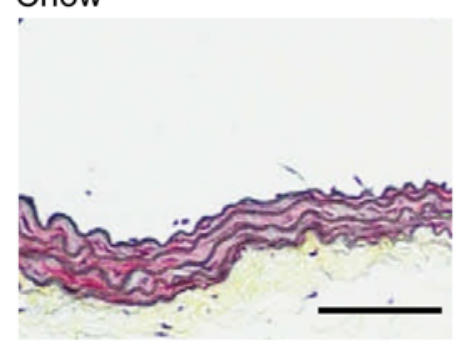

DDC

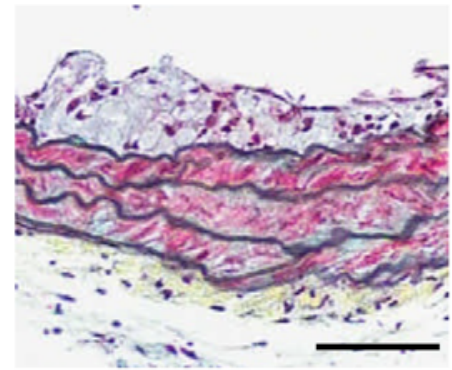

G Representative BCA stained for Mac-2 and SM $\alpha$-actin

Mac-2

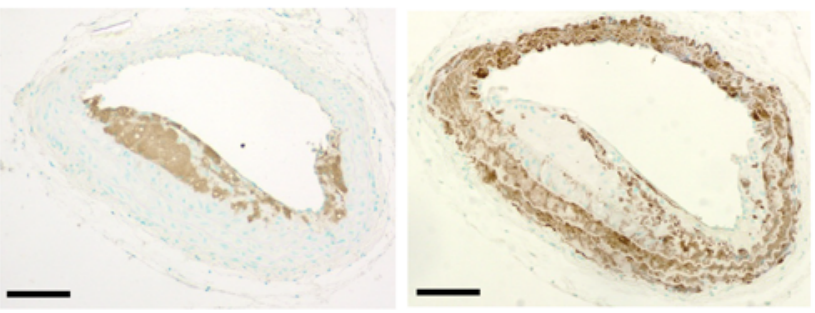

H Representative BCA showing intraplaque hemorrhage

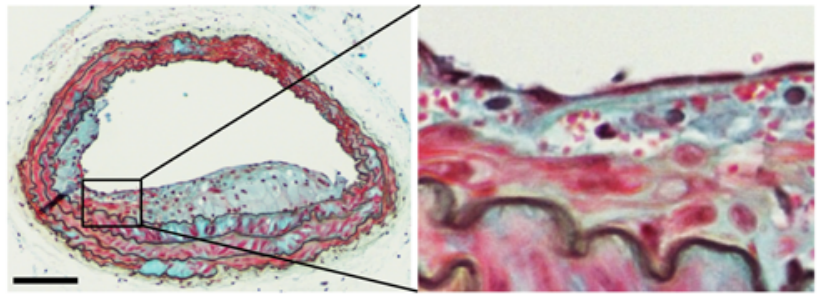

\section{Chow}

C BCA max lesion area

D BCA medial area
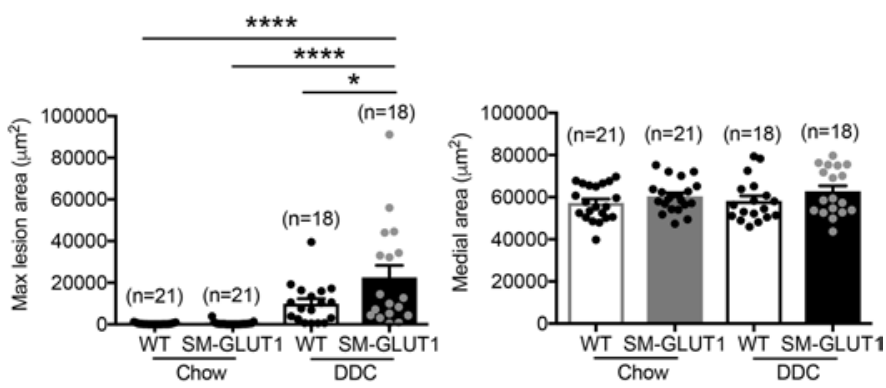

E

Macrophage area

F $\mathrm{SM} \alpha$-actin area

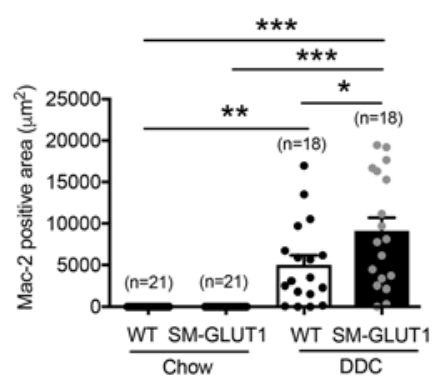

I

Necrotic cores

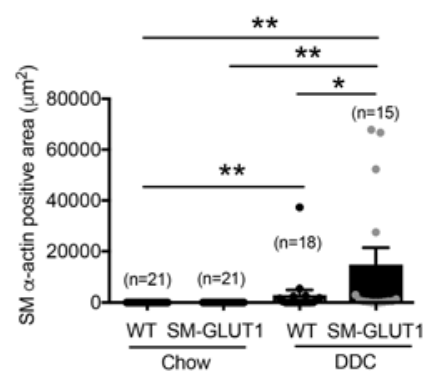

$\mathbf{J}$ Intraplaque hemorrhage
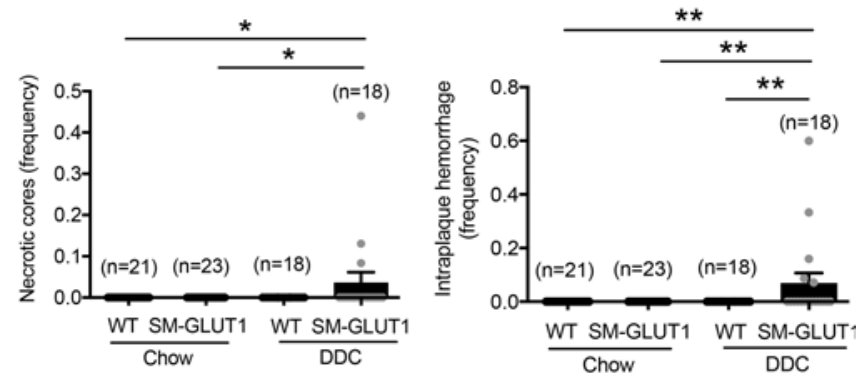

Figure 5. Smooth muscle-targeted GLUT1 overexpression increases BCA lesion size and complexity in DDC-fed mice. BCA lesion morphology (A and B) was assessed in serial sectioned BCAs stained by the Movat's pentachrome stain. Arrow indicates a necrotic core in a BCA from a DDC-fed SM-CLUT1 mouse. BCA morphology was assessed in all chow-fed mice and in DDC-fed mice with plasma cholesterol levels above 1,000 mg/dl. BCA maximal lesion area (C), medial area (D), Mac-2-positive lesion area (E), and SM $\alpha$-actin-positive lesion area (F). (G) Representative lesion cross-sections adjacent to the maximal lesion site stained for Mac-2 and SM $\alpha$-actin. (H) Representative lesion from a DDC-fed SM-GLUT1 mouse showing intraplaque hemorrhage. Frequency of necrotic cores (I) and intraplaque hemorrhage (J). Results are expressed as mean \pm SEM $(n=15-23)$. Statistical analysis was performed by 1-way ANOVA with Tukey's post hoc tests or by Kruskal-Wallis test with Dunn's post hoc tests (I and J). One animal was excluded from the SM-GLUT1 chow group as a statistical outlier by Grubbs' test. ${ }^{*} P<0.05$; ${ }^{* *} P<0.01 ;{ }^{* *} P<0.001 ;{ }^{* * *} P<0.0001$. Scale bars: $100 \mu \mathrm{m}$

BM transplants, with GLUT1 overexpression in myeloid cells controlled under the CD68 promoter or controls, using a stem cell retroviral transduction protocol previously described (14) and shown in Figure 6A. At the end of the study, isolated BM-derived macrophages (BMDMs) from these mice took up more glucose and exhibited increased lactate release (Figure 6, B and C), demonstrating that GLUT1 overexpression resulted in functional effects. However, GLUT1 overexpression in myeloid cells did not promote atherosclerosis in chow-fed mice or those fed DDC (Figure 6D). As in DDC-fed SM-GLUT1 
A Study design

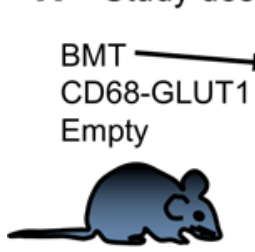

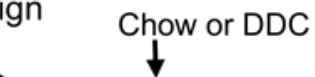

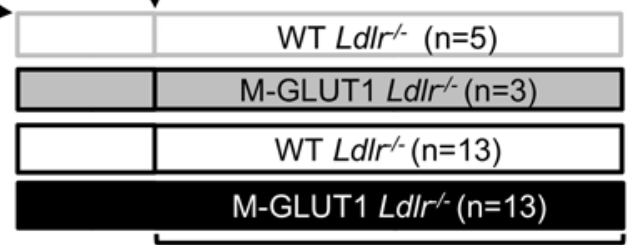

7 weeks recovery

D Aortic lesion area M-GLUT1

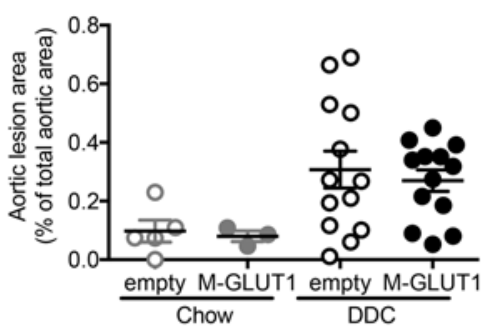

G GTT M-GLUT1

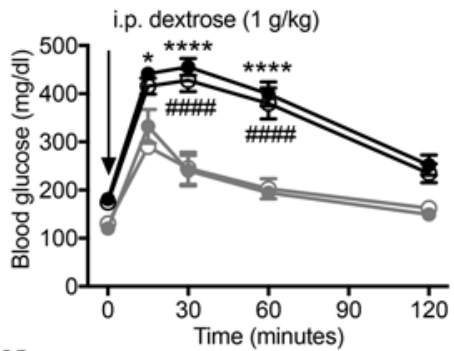

K Body weight LFD-fed SM-GLUT1

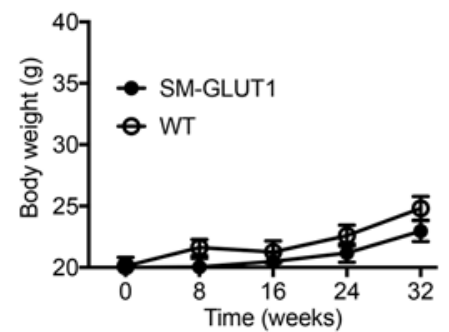

16 weeks

E Cholesterol M-GLUT1

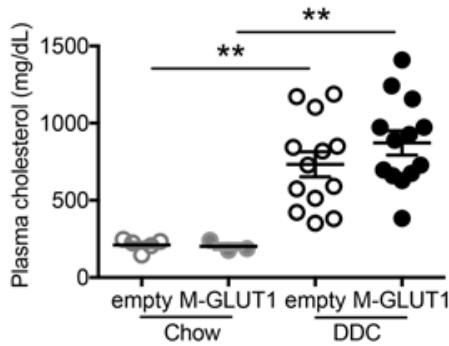

B 2-DOG uptake M-GLUT1

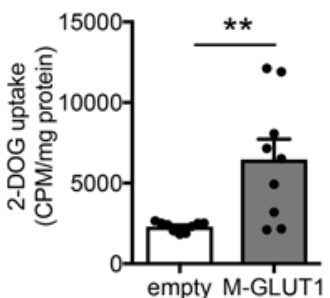

C Lactate release M-GLUT1

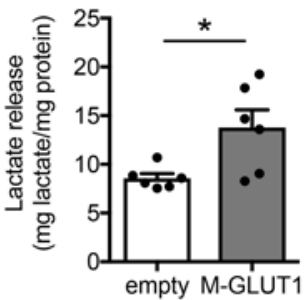

F Triglycerides M-GLUT1

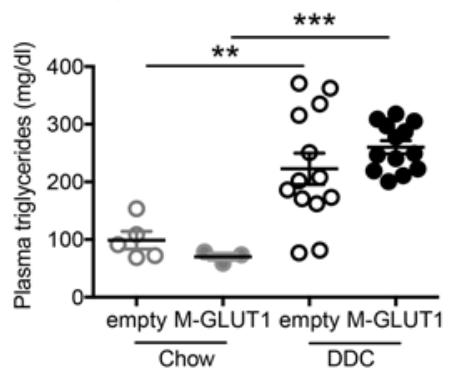

H Body weight M-GLUT1 I Cholesterol LFD-fed J Triglycerides LFD-fed

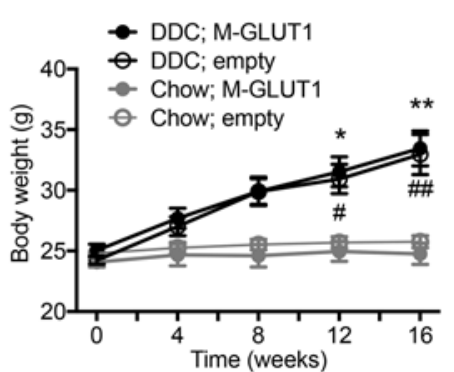

L Aortic lesion area LFD-fed SM-GLUT1
SM-GLUT1

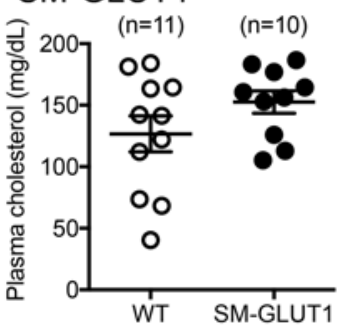

M BCA lesion WT
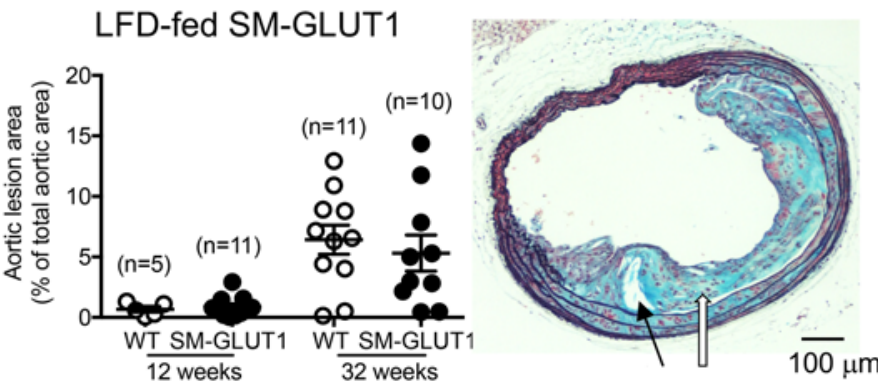
SM-GLUT1

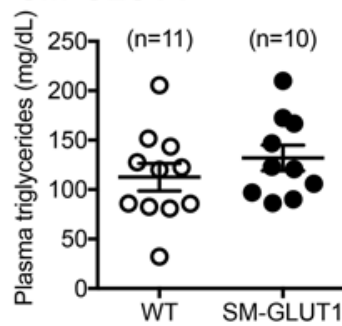

N BCA lesion SM-GLUT1

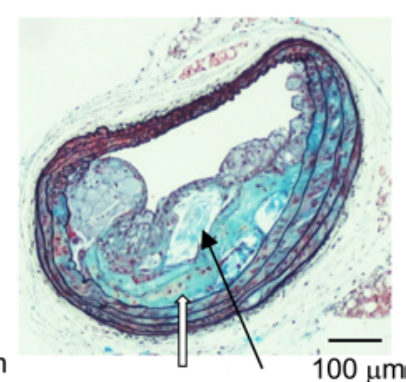

Figure 6. Neither GLUT1 overexpression in myeloid cells nor smooth muscle-targeted GLUT1 overexpression in low-fat diet-fed mice increases atherosclerosis. CD68-GLUT1 overexpressing male Ldl/ ${ }^{-/-}$mice (A) exhibited increased 2-DOG uptake (B) and lactate release (C) in BM-derived macrophages, versus littermate controls ( $n=9$ in B; $n=6$ in C). Myeloid cell GLUT1 overexpression did not affect aortic atherosclerosis (D), plasma cholesterol (E), triglycerides (F), glucose intolerance (G), or body weight (H). Results are expressed as mean \pm SEM. Female Ldlr-/- SM-GLUT1 mice and littermate controls were fed a low-fat semipurified diet (LFD) for 12-32 weeks. Plasma cholesterol (I), triglycerides (J), and body weight (K) were lower than in DDC-fed mice. Mice exhibited aortic lesions, but SM-GLUT1 had no effect (L). ( $\mathbf{M}$ and $\mathbf{N}$ ) BCA lesions were advanced, with necrotic cores (black arrows) and fibrotic areas (white arrows). Results are expressed as mean \pm SEM. Statistical analysis was performed by 1-way ANOVA (B, C, E, F); 2-way ANOVA

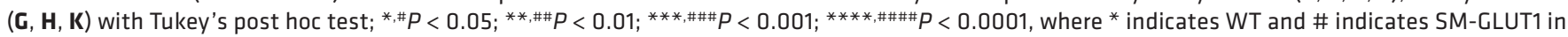
G and H. BMT, bone marrow transplant; LFD, low-fat diet.

mice, DDC feeding resulted in a metabolic syndrome phenotype, characterized by increased plasma cholesterol, triglycerides, impaired glucose tolerance, and obesity (Figure 6, E-H), without an effect of myeloid cell GLUT1 overexpression. These results suggest that the role of increased glucose metabolism in accelerating progression of atherosclerosis in SMCs is selective to this cell type, at least as compared with myeloid cells. 
We next investigated whether the metabolic syndrome phenotype is required for the proatherogenic effect of SM-GLUT1 overexpression. LDLR-deficient mice fed a semipurified low-fat diet (34) exhibit atherosclerosis at 12-32 weeks. We therefore conducted an additional study in this model to assess the requirement for GLUT1-mediated effects in SMCs. Female $L d l r^{--}$WT and $L d l r^{\prime-}$ SM-GLUT1 mice were fed the semipurified low-fat diet, starting at 8-12 weeks of age for 12 or 32 weeks. As expected, this diet did not increase body weight beyond normal effects seen with age, and plasma cholesterol and triglycerides remained low (Figure 6, I-K). Thus, animals had plasma cholesterol levels in the range of 100-200 mg/ dl. Mice began to develop aortic lesions at 12 weeks after diet switch from chow to the low-fat diet, with substantial aortic lesions present after 32 weeks (Figure 6L). The BCA lesions showed an advanced lesion phenotype, with fibrotic areas and the presence of necrotic cores (Figure 6, M and N). Unlike mice fed DDC, GLUT1 overexpression in SMCs did not influence lesion size at either of the time points investigated (Figure 6L). These results suggest that the proatherogenic effect of smooth muscle GLUT1 overexpression is selective to mice fed DDC to induce a metabolic syndrome phenotype.

GLUT1 does not appear to directly drive SMC proliferation or hyaluronan synthesis. We next performed a series of experiments to evaluate the mechanism whereby smooth muscle GLUT1 overexpression synergizes with DDC feeding in promoting atherosclerosis. With the increased SMC content only in lesions of DDC-fed SM-GLUT1 mice (Figure 5F and Supplemental Table 2), we first investigated if GLUT1 expression in SMCs was necessary or sufficient for proliferation of these cells. Total aortic mRNA from SM-GLUT1 mice following the 16-week DDC study suggest a small (nonsignificant) increase in the cell proliferation marker cyclin D1 (gene Ccnd1) in SM-GLUT1 mice (Supplemental Figure 2A), leaving the possibility open for localized effects of GLUT1 overexpression on proliferation. To assess the relationship between Slc2a1 and $C$ cnd 1 expression, we took advantage of the ability of SMCs to change from a contractile phenotype in vivo to a synthetic proliferative phenotype when placed in culture (35). This phenotypic change is also believed to occur in atherosclerotic lesions (20). Indeed, mouse aortic SMCs exhibited a dramatic increase in Cond1 mRNA when expanded in culture for 9 days (Supplemental Figure 2B). However, the change in proliferative capacity was not accompanied by a corresponding increase in Slc2a1 mRNA expression (Supplemental Figure 2C), suggesting that upregulation of GLUT1 is not required for SMC proliferation. We further investigated if overexpression of GLUT1 could induce proliferation in cultured SMCs. GLUT1 was overexpressed in isolated aortic SMCs using a retrovirus. Transduction of SMCs with this retrovirus resulted in a marked increase in Slc2al mRNA, as compared with SMCs transduced with the empty pBM vector control (Supplemental Figure 2D), but did not increase Ccnd1 mRNA levels and, rather, resulted in a small reduction in Ccnd1 mRNA (Supplemental Figure 2E). These data argue that GLUT1 expression in SMCs is independent of the proliferative status of the cells. The results were corroborated by immunohistological evaluation of SMC proliferation, using the cell proliferation marker Ki-67. There was no significant change in Ki-67 staining in BCA cross-sections of DDC-fed SM-GLUT1 mice when normalized to either medial or lesion area, as compared with DDC-fed WT mice, although there was marked variation between mice, probably due to differences in lesional stage (Supplemental Table 2). Thus, the increase in atherosclerotic progression in DDC-fed SM-GLUT1 mice is unlikely to be due to direct effects of GLUT1 on SMC proliferation. Furthermore, there was no significant effect of SM-GLUT1 expression on SMC apoptosis, measured through TUNEL staining of BCA cross-sections (data not shown).

Increased smooth muscle hyaluronan synthesis has been shown to drive atherosclerosis in $A p o e^{-/-}$mice (36), and we observed an increase in gene expression of Has1 and Has3 in DDC-fed SM-GLUT1 mice (Figure 4, I and $\mathrm{J}$ ). We therefore stained lesions for the presence of hyaluronan by using a biotinylated hyaluronan-binding protein (37). BCA medial levels of hyaluronan were increased in DDC-fed SM-GLUT1 mice, as compared with chow-fed mice, whereas no significant increase was detected in lesional hyaluronan (Supplemental Figure 2, F and G). We next investigated if GLUT1 expression is sufficient to increase hyaluronan secretion. However, GLUT1 overexpression in SMCs did not result in increased secretion of hyaluronan (Supplemental Figure 2H). These results are consistent with the lack of GLUT1 on intermediates in the glycosamine pathway. It is therefore not clear that changes in hyaluronan observed in this model contribute directly to lesion progression.

Increased GLUT1 facilitates SMC cytokine production through glycolysis and the polyol pathway. We next investigated if SM-GLUT1 overexpression in DDC-fed mice alters the inflammatory phenotype of the aorta. Inflammation is increasingly recognized as an integral process of atherosclerosis in mouse models and humans $(9,10)$, and SM-GLUT1 mice on a C57BL/ 6 background have previously been shown to exhibit elevated immunostaining for CCL2 following vascular injury (24). Gene expression data from 
A Aortic Tnfa

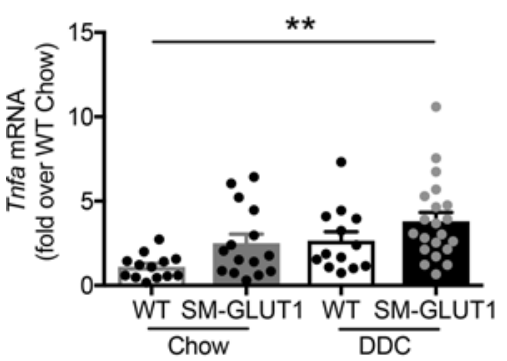

D $\mathrm{SMC} \mathrm{CCl} 2$

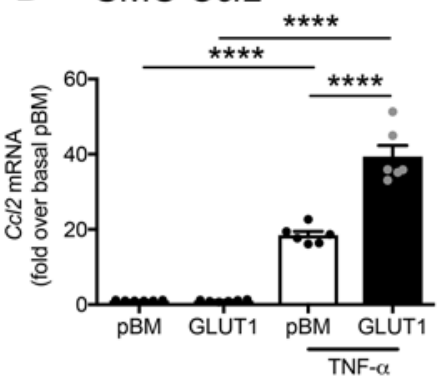

G $\mathrm{SMC} \mathrm{Ccl} 2$

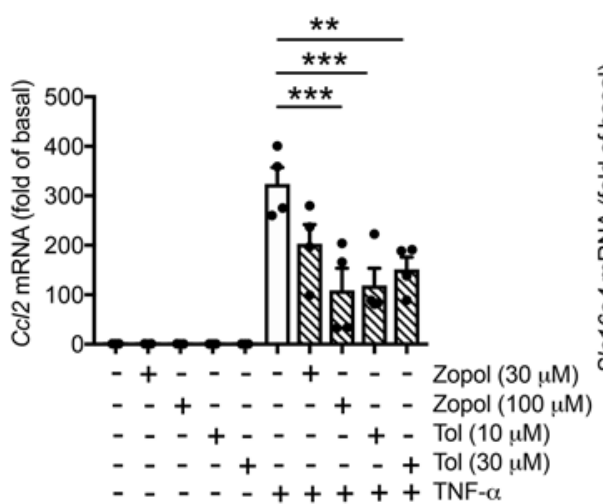

B Aortic $\mathrm{Ccl} 2$

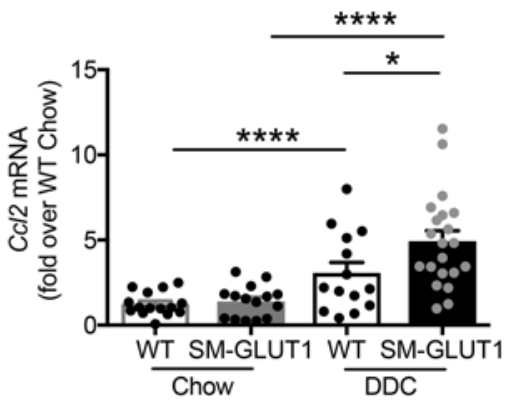

E SMC CCL2 secretion

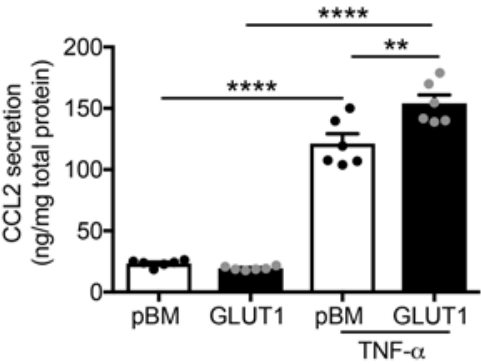

H SMC Slc16a4

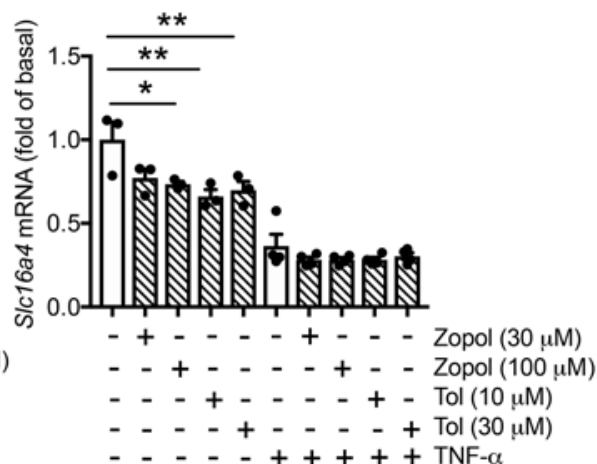

c SMC Tnfa

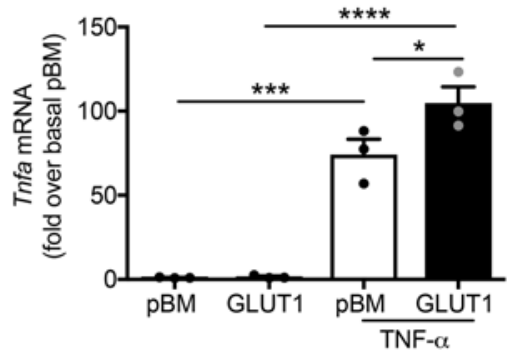

F $\mathrm{SMC} \mathrm{CCl} 2$

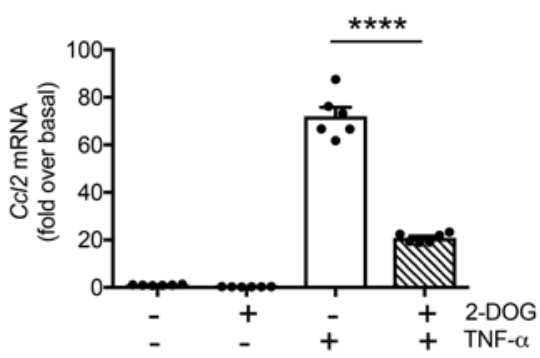

I SMC S/c2a1

Figure 7. GLUT1 overexpression synergizes with TNF- $\alpha$ to promote CCL2 and TNF- $\alpha$ production in aortic SMCs. (A and B) Aortas from SM-CLUT1 mice exhibit increased expression of Tnfa and Ccl2 mRNA ( $n=15$ for WT chow and SM-GLUT1 chow groups; $n=14$ for WT DDC group; $n=21$ for SM-GLUT1 DDC group). Mouse aortic SMCs transduced with a retrovirus to overexpress GLUT1, or the empty pBM virus as control, were stimulated with mouse recombinant TNF- $\alpha(5 \mathrm{ng} / \mathrm{ml})$ for 1 hour (C) or 24 hours (D-I). Tnfa (C) and Cc/2 mRNA (D, F, G) were measured by real-time PCR. TNF- $\alpha$-driven Cc/2 mRNA and CCL2 secretion, measured by ELISA, in SMCs was increased by GLUT1 overexpression ( $D$ and $\mathbf{E}$ ). TNF- $\alpha$ induction of $C c / 2$ mRNA levels is prevented by inhibiting glycolysis by incubation of the cells in the presence of $1 \mathrm{mM}$ 2-DOG (F) or by inhibiting aldose reductase by 2 different inhibitors (zopolrestat [zopol] or tolrestat [tol]) in the polyol pathway (G). (H) TNF- $\alpha$-induced suppression of SIc16a4 was not altered by aldose reductase inhibitors. (I) TNF- $\alpha$-induced SIc2a1 was not altered by aldose reductase inhibitors. Results are expressed as mean \pm SEM ( $n=3$ in $\mathbf{C} ; n=6$ in $\mathbf{D}-\mathbf{F} ; n=3-4$ in G-I). Statistical analysis was performed by 1-way ANOVA with Tukey's post hoc test; ${ }^{*} P<0.05 ;{ }^{* *} P<0.001 ;{ }^{* * *} P<0.0001$.

whole aortas demonstrated an effect of smooth muscle GLUT1 overexpression on enhancing expression of some cytokines and chemokines in DDC-fed mice. Thus, DDC-fed SM-GLUT1 mice exhibited increased aortic Tnfa mRNA levels, as compared with WT chow-fed mice (Figure 7A). Levels of the chemokine $C c 12$ were increased by DDC-feeding and were further increased in SM-GLUT1 mice fed DDC (Figure 7B). To assess if SMCs (as opposed to other lesional cell types) were directly producing the increased cytokines, we quantified Tnfa and $C c l 2$ mRNA in primary cultured aortic SMCs (Figure 7, C and D) in which GLUT1 was overexpressed in the presence and absence of TNF- $\alpha$. The increase in GLUT1 expression in isolated SMCs promoted increased Tnfa mRNA levels 1 hour after stimulation with TNF- $\alpha$ (Figure 7C). Similarly, GLUT1 overexpression enhanced the stimulatory effect of TNF- $\alpha$ 
on Ccl2 mRNA after 24 hours (Figure 7D). GLUT1 overexpression had no effect in the absence of TNF- $\alpha$, suggesting that GLUT1 overexpression does not directly drive cytokine secretion by SMCs but can facilitate a cytokine response with additional inflammatory stimuli. Consistently, TNF- $\alpha$ stimulation led to enhanced CCL2 release in isolated SMCs treated for 48 hours, and this effect was enhanced in SMCs overexpressing GLUT1 (Figure 7E).

To determine if enhanced glycolysis is required for TNF- $\alpha$ induction of Ccl2 mRNA, we treated SMCs with TNF- $\alpha$ in the presence or absence of 2-DOG, which inhibits glycolysis at several early steps. 2-DOG markedly prevented the effect of TNF- $\alpha$ on $C c l 2$ induction (Figure 7F). Furthermore, 2 different aldose reductase inhibitors, zopolrestat and tolrestat, also prevented the effect of TNF- $\alpha$ on $C c l 2$ induction (Figure $7 G$ ), indicating a role for the polyol pathway in TNF- $\alpha$-induced CCL2 production. Conversely, elevating levels of succinate, by using cell-permeable diethyl succinate or diethyl butyl malonate, did not enhance the effects of TNF- $\alpha$ on $C c l 2$ (data not shown). Thus, our data demonstrate that increased glucose flux through GLUT1 enhances a positive feedback mechanism that allows TNF- $\alpha$ to induce its own production, as well as that of CCL2. Because lactate has recently been shown to promote a phenotypic switch in SMCs differentiated from human induced pluripotent stem cells (a switch that was dependent on expression of the monocarboxylic acid transporter MCT4 [Slc16a4]; ref. 38), we analyzed Slc16a4 mRNA levels in SMCs stimulated with TNF- $\alpha$ in the presence and absence of aldose reductase inhibitors. Aldose reductase inhibitors slightly suppressed Slc16a4 mRNA levels in the absence of TNF- $\alpha$, and TNF- $\alpha$ stimulation resulted in an additional suppression, which was not affected by aldose reductase inhibitors (Figure $7 \mathrm{H}$ ). Consistently, MCT4 levels have been shown to be reduced by IL-1 $\beta$ in endothelial cells (39). TNF- $\alpha$-induced GLUT1 also was not altered by aldose reductase inhibitors (Figure 7I). These results suggest that the aldose reductase pathway selectively enhances TNF- $\alpha$-induced CCL2 production.

DDC-feeding increases circulating levels of CCR2-positive Ly6Chi monocytes. The synergistic effect of GLUT1 overexpression in SMCs toward accelerating atherosclerosis was limited to mice with a metabolic syndrome phenotype. CCR2, the receptor for CCL2, is known to be highly expressed on the Ly6 $\mathrm{C}^{\mathrm{hi}}$ monocyte subset, which readily enters lesions of atherosclerosis through CCR2 (7). Consistently, real-time PCR of sorted Ly $6 \mathrm{C}^{\text {hi }}$ and $\mathrm{Ly} 6 \mathrm{C}^{\text {lo }}$ blood monocytes from chow-fed and DDC-fed mice showed much higher levels of $C c r 2$ mRNA in Ly $6 C^{\text {hi }}$ monocytes than in Ly6 $\mathrm{C}^{\text {lo }}$ monocytes in all groups of mice (Supplemental Figure 3A). SM-GLUT1 expression did not alter blood monocyte levels of $C$ cr2 or levels of Ly6 $\mathrm{Ch}^{\text {hi }}$ or Ly6C $\mathrm{C}^{\text {lo }}$ monocytes (Supplemental Figure 3, A and B).

We again used flow cytometry (Supplemental Figure 3, C and D) to determine whether DDC feeding leads to a shift in the leukocyte profile or altered levels of cell surface CCR2 on monocyte subsets. The results demonstrate that DDC feeding induced a transient early increase in levels of blood neutrophils at 4 weeks, followed by monocytosis (Table 1). Elevated Ly6C $\mathrm{C}^{\text {hi }}$ monocytes persisted after 12 weeks on the diet. In addition, cell-surface CCR2 expression in the $\mathrm{Ly} 6 \mathrm{C}^{\text {hi }}$ monocyte population was significantly elevated in DDC-fed mice throughout the 12-week study (Table 1). Conversely, lean mice fed the low-fat semipurified diet did not show consistently elevated levels of monocytes or increases in cell surface CCR2 (Table 1). Thus, DDC feeding is likely to promote atherosclerosis, in part, by causing increased circulating levels of CCR2-expressing monocytes with a high ability to enter lesions of atherosclerosis.

Smooth muscle GLUT1 overexpression increases Ly6Chi monocyte recruitment to lesions of atherosclerosis. Finally, to confirm that the increased CCL2 production in GLUT1-expressing SMCs can lead to increased Ly6 $\mathrm{C}^{\mathrm{hi}}$ monocyte recruitment to lesions in vivo, we performed a monocyte tracing experiment. Mice overexpressing GLUT1 in SMCs and WT littermates were fed DDC for 16 weeks to induce atherosclerotic lesions and a metabolic syndrome phenotype. Monocytes were then depleted by chlodronate injection, and Ly6 $\mathrm{C}^{\mathrm{hi}}$ monocytes were labeled by fluorescent microbeads (Figure 8A). Effective blood monocyte depletion was demonstrated by flow cytometry 16 hours after chlodronate injection (Figure 8B). Four days after depletion, a clear majority of blood monocytes were Ly6C $\mathrm{C}^{\mathrm{h}}$ monocytes (Figure $8 \mathrm{C}$ ). Approximately $15 \%$ of blood monocytes were bead-labeled, and the labeling efficiency was similar in DDC-fed mice with and without SM-GLUT1 expression (Figure 8D). Labeled monocyte recruitment to the BCA was quantified on cross-sections immunostained for macrophages. Mice with SMC GLUT1 overexpression exhibited an increased recruitment of monocytes to these BCA lesions (Figure 8, E and F). These studies confirm that smooth muscle glucose metabolism increases monocyte recruitment to lesions in this mouse model of metabolic syndrome. 
Table 1. Effect of metabolic syndrome phenotype on WBC populations and monocyte CCR2 levels

\begin{tabular}{|c|c|c|c|}
\hline Cell population & Chow, 4 w $(n=8)$ & DDC, 4 w $(n=7)$ & $P$ value \\
\hline Total WBC (cells/ $\mu$ l) & $9,378 \pm 1,293$ & $10,866 \pm 1,126$ & ns \\
\hline Neutrophils (cells/ $\mu \mathrm{l}$ ) & $1,450 \pm 279$ & $2,375 \pm 265$ & 0.033 \\
\hline Ly6Chi (cells/ $\mu$ l) & $439 \pm 66$ & $486 \pm 58$ & ns \\
\hline Ly6C hi CCR2 (MIF) & $1,086 \pm 90$ & $1,386 \pm 80$ & 0.028 \\
\hline 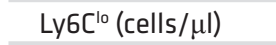 & $256 \pm 33$ & $324 \pm 51$ & ns \\
\hline Total WBC (cells/ $\mu$ l) & $7,138 \pm 752$ & $12,091 \pm 1,188$ & 0.003 \\
\hline Neutrophils (cells/ $\mu \mathrm{l}$ ) & $1,272 \pm 234$ & $1,405 \pm 126$ & ns \\
\hline Monocytes (cells/ $\mu$ l) & $614 \pm 56$ & $1,049 \pm 90$ & 0.001 \\
\hline Ly6C hi (cells/ $\mu \mathrm{l})$ & $324 \pm 29$ & $535 \pm 54$ & 0.003 \\
\hline Ly6C hi CCR2 (MIF) & $1,132 \pm 76$ & $1,863 \pm 61$ & $<0.001$ \\
\hline Total WBC (cells/ $\mu$ l) & $15,200 \pm 1,699$ & $17,095 \pm 2,051$ & ns \\
\hline Neutrophils (cells/ $\mu \mathrm{l}$ ) & $2,403 \pm 179$ & $1,909 \pm 244$ & ns \\
\hline Monocytes (cells/ $\mu l)$ & $802 \pm 83$ & $1,207 \pm 182$ & 0.047 \\
\hline Ly6Chi (cells/ $\mu \mathrm{l})$ & $520 \pm 45$ & $815 \pm 130$ & 0.033 \\
\hline Ly6C hi CCR2 (MIF) & $5,041 \pm 242$ & $6,171 \pm 364$ & 0.020 \\
\hline 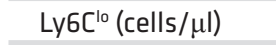 & $282 \pm 44$ & $391 \pm 57$ & ns \\
\hline Ly6C ${ }^{10}$ CCR2 (MIF) & $490 \pm 34$ & $383 \pm 47$ & ns \\
\hline Cell population & Chow, 4 w $(n=8)$ & LFD, 4 w $(n=10)$ & $P$ value \\
\hline Total WBC (cells/ $\mu$ l) & $13,075 \pm 693$ & $13,137 \pm 1,025$ & ns \\
\hline Neutrophils (cells/ $\mu \mathrm{l}$ ) & $2,076 \pm 508$ & $1,444 \pm 117$ & ns \\
\hline Monocytes (cells/ $\mu l)$ & $655 \pm 67$ & $674 \pm 73$ & ns \\
\hline Monocytes (cells/ $\mu l$ ) & $907 \pm 62$ & $1,039 \pm 98$ & ns \\
\hline Ly6Chi (cells/ $\mu$ l) & $577 \pm 39$ & $666 \pm 67$ & ns \\
\hline Ly6C hi CCR2 (MIF) & $5,722 \pm 275$ & $7,000 \pm 171$ & 0.001 \\
\hline 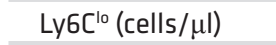 & $330 \pm 29$ & $373 \pm 34$ & ns \\
\hline Ly6C ${ }^{10}$ CCR2 (MIF) & $1,110 \pm 72$ & $1,210 \pm 62$ & ns \\
\hline Cell population & Chow, $12 \mathrm{w}(n=8)$ & LFD, 12 w $(n=9)$ & $P$ value \\
\hline 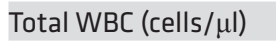 & $6,535 \pm 556$ & $7,559 \pm 958$ & ns \\
\hline Neutrophils (cells/ $\mu$ l) & $1,521 \pm 95$ & $2,062 \pm 213$ & 0.042 \\
\hline Monocytes (cells/ $\mu$ l) & $486 \pm 50$ & $672 \pm 110$ & ns \\
\hline Ly6Chi (cells/ $\mu$ l) & $321 \pm 33$ & $432 \pm 39$ & 0.041 \\
\hline Ly6C hi CCR2 (MIF) & $722 \pm 78$ & $864 \pm 99$ & ns \\
\hline 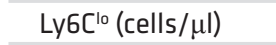 & $165 \pm 18$ & $188 \pm 53$ & ns \\
\hline Ly6C ${ }^{10}$ CCR2 (MIF) & $200 \pm 27$ & $239 \pm 20$ & ns \\
\hline
\end{tabular}

Male $\mathrm{Ldll}^{-1-}$ mice were fed regular chow, a low-fat semipurified diet (LFD), or DDC for 4-12 weeks. Leukocyte populations and CCR2 cell-surface levels were determined by flow cytometry. Results are expressed as mean \pm SD; MFI, mean fluorescence intensity. W, weeks.

\section{Discussion}

Our results demonstrate that increased GLUT1 expression in SMCs promotes atherosclerosis in the presence of elevated levels of CCR2-positive blood monocytes in a mouse model of metabolic syndrome, and suggest a potentially novel mechanism whereby arterial glucose metabolism synergizes with metabolic syndrome to 
A Study design

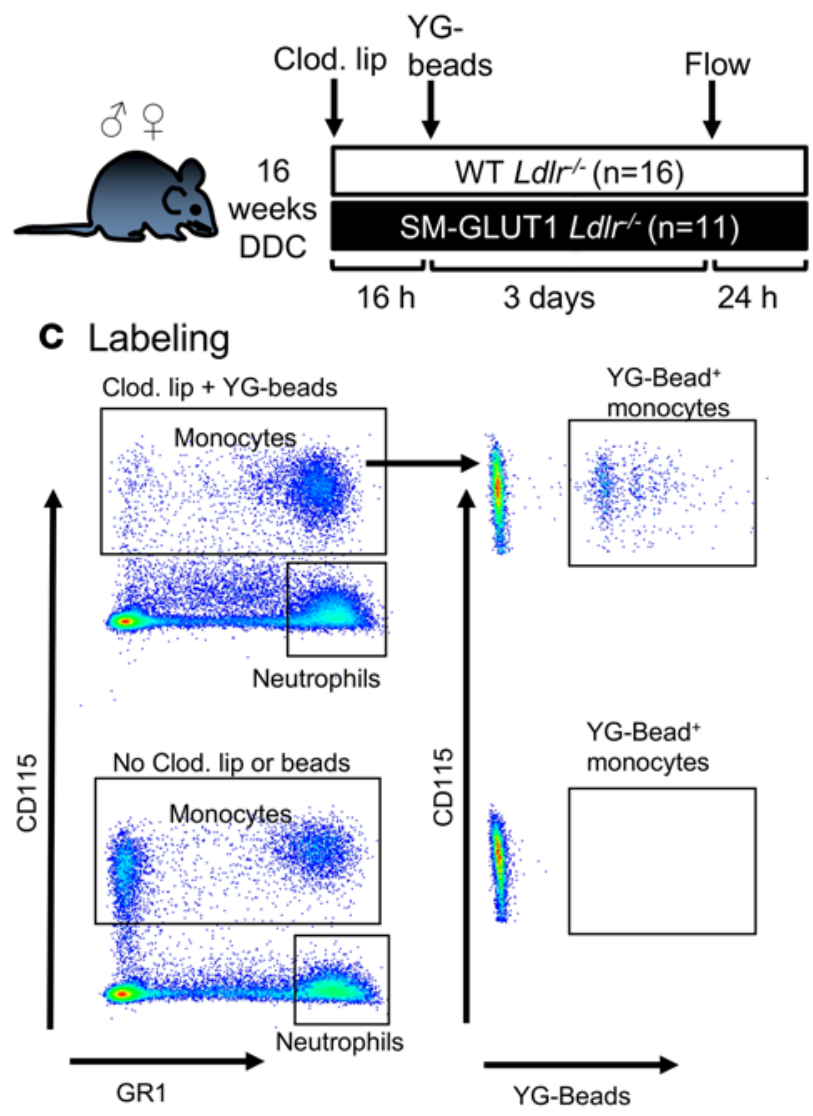

E Monocyte recruitment to BCA

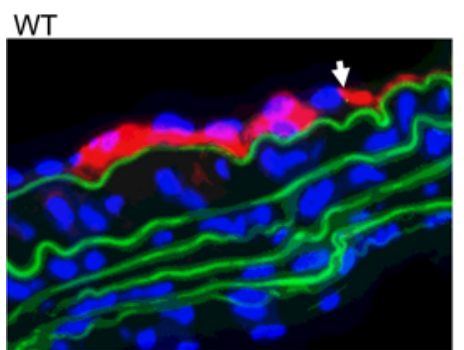

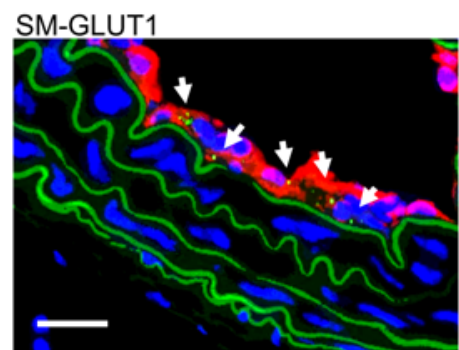

B Depletion

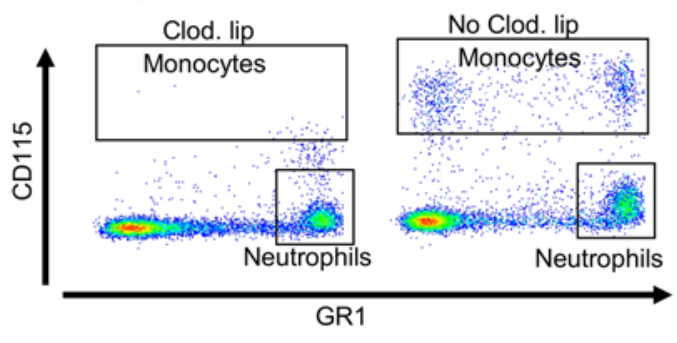

D Labeling efficiency
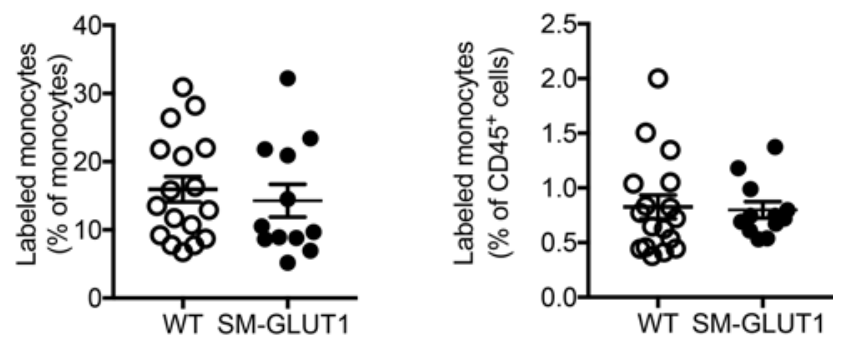

\section{F Monocyte recruitment to $\mathrm{BCA}$}
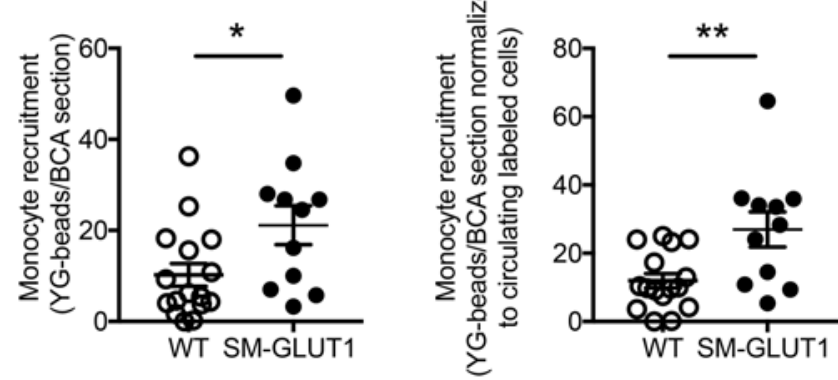

Figure 8. SMC GLUT1 promotes monocyte recruitment in vivo. (A) Study design. WT and SM-GLUT1 mice were fed DDC for 16 weeks before monocytes were depleted with clodronate liposomes (Clod. lip). Sixteen hours after clodronate injection, yellow-green (YG) microparticles were injected retro-orbitally to label newly formed monocytes (predominantly Ly6C ${ }^{\text {hi }}$ monocytes). Three days after YG-bead injection, labeling efficiency was determined by flow cytometry. The experiment was terminated 4 days after labeling. (B) Flow cytometric analysis of the level of depletion of monocytes in the blood (>98\%) in a Clod. lipinjected mouse compared with noninjected mouse. (C) Flow cytometric analysis of labeling efficiency in the circulation in a Clod. lip- and YG-bead-injected mouse compared with mouse not injected with Clod. lip or YG-beads. (D) Labeling efficiency as \% of total monocytes (Ly6C ${ }^{\text {hi }}$ monocytes constitutes $>80 \%$ of all monocytes 4 days after depletion), and as \% of total CD45+ cells. (E) Representative images from brachiocephalic artery (BCA). Green, YG-beads (white arrows) and elastic lamina; red, Mac-2 (macrophages); blue, DAPI (nuclei). (F) Quantification of YG-beads/BCA with and without normalizing to percent of labeled monocytes. $n=11-17$. Statistical analysis was performed by 2 -tailed unpaired Student's $t$ test. ${ }^{*} P<0.05 ;{ }^{* *} P<0.01$.

accelerate atherosclerosis. The notion that local processes, including inflammation in the lesional environment, play an important role in CVD risk in humans is gaining increased traction (6). A large number of animal studies also support this concept. Although lesional macrophages are generally believed to produce most of the inflammatory mediators in atherosclerotic lesions, SMCs produce chemokines and cytokines when exposed to an inflammatory environment, as demonstrated in this study and others (40-44). SMCs readily respond to IL-1 $\beta$ and TNF- $\alpha$, as well as other cytokines, and are able to produce and secrete cytokines and chemokines. These cells are therefore likely to contribute to lesional leukocyte recruitment and inflammation 


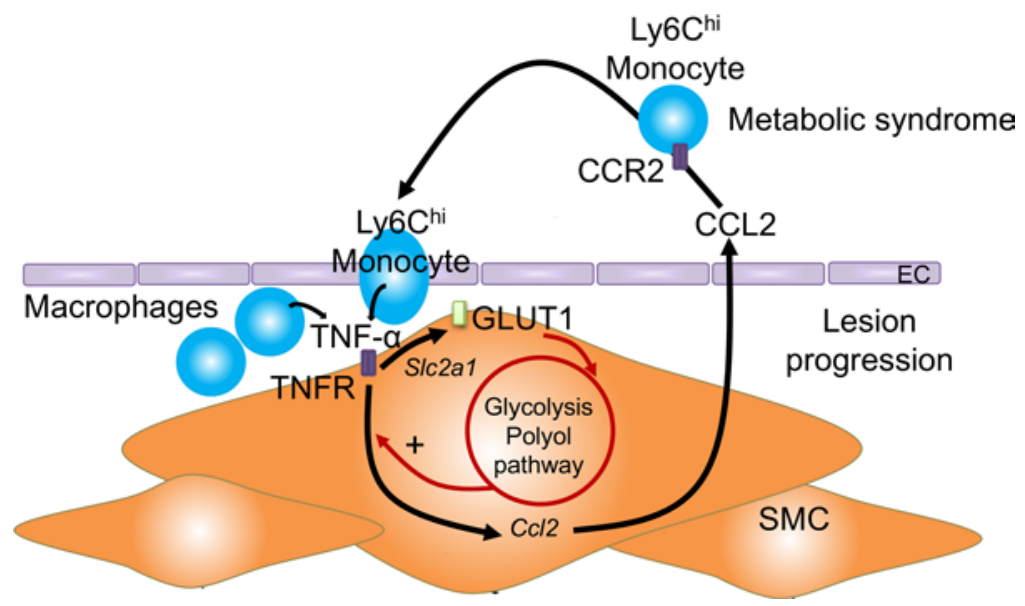

Figure 9. Mechanistic model. The metabolic syndrome phenotype results in monocytosis and increased numbers of circulating CCR2-positive Ly6C ${ }^{\text {hi }}$ monocytes. These monocytes are recruited to forming lesions of atherosclerosis. In the lesion, monocytes and mature macrophages secrete cytokines, including TNF- $\alpha$. TNF- $\alpha$, in turn, acts on adjacent SMCs to induce GLUT1 and the chemokine CCL2. Increased GLUT1 stimulates glycolysis and the polyol pathway in the SMCs. The polyol pathway, through aldose reductase, enhances TNF- $\alpha$-induced CCL2 expression and secretion. SMC-derived CCL2 further stimulates Ly6Chi monocyte recruitment to the lesion, creating a cycle driving lesion progression in the setting of metabolic syndrome. EC, endothelial cell; TNFR, TNF receptor.

in a positive feed-forward mechanism. Our data support a role for SMCs in promoting lesional inflammation. Furthermore, we show that, in addition to promoting TNF- $\alpha$ and CCL2 production by SMCs, TNF- $\alpha$ also induces GLUT1 expression, leading to increased glucose uptake, glycolysis, and polyol pathway activation in these cells. Thus, the inflammatory lesion environment changes SMC metabolism by increasing glucose uptake and aerobic glycolysis. Consistently, we show that SMCs express increased levels of GLUT1 when in close proximity to a lesion of atherosclerosis.

What is the functional significance of the increased SMC glucose uptake induced by inflammation? We investigated if increased smooth muscle GLUT1 expression near atherosclerotic lesions could be causative of disease by generating a transgenic $L d l r^{-1-}$ mouse with SMC-targeted GLUT1 overexpression. Our results demonstrate that increased SMC GLUT1 expression does not induce atherogenesis in mice fed regular chow or low-fat diets, but it strikingly increases atherosclerosis in a mouse model of metabolic syndrome. SMC expression of GLUT1 also resulted in more advanced lesions, characterized by increased free cholesterol levels with regard to cholesteryl ester levels. The ratio of free cholesterol/cholesteryl esters has been shown to increase as the lesion becomes more advanced (45).

We further show that the ability of increased SMC GLUT1 expression to enhance atherosclerosis is unlikely to be due to direct effects of GLUT1 on SMC accumulation or hyaluronan production. Instead, GLUT1 overexpression enhanced the inflammatory phenotype of the artery wall and of isolated SMCs, augmenting the ability of these cells to secrete the chemokine CCL2 and stimulating monocyte recruitment and macrophage accumulation. The effect of SM-GLUT1 expression on atherosclerosis is associated with an increased aortic expression of CCL2 and increased levels of circulating CCR2-positive Ly6C $\mathrm{C}^{\text {hi }}$ monocytes. This monocyte population readily enters lesions of atherosclerosis through a process dependent on the CCL2 receptor CCR2 $(7,46)$. In support, human studies find that increased monocyte populations expressing CCR2 are predictive of CVD (47). Consistent with the role for metabolic syndrome in synergizing with SMC GLUT1, mice fed a low-fat diet showed no consistent increase in circulating CCR2-positive Ly6 $\mathrm{C}^{\mathrm{hi}}$ monocytes, and SMC GLUT1 expression did not promote atherosclerosis in these mice.

Cholesterol accumulation promotes myelopoiesis by stimulating hematopoietic BM progenitor cell proliferation (48). Other studies have shown a strong positive correlation between circulating monocyte levels and total plasma cholesterol levels in different animal models and in humans (49-51). It is therefore likely that DDC feeding increases monocyte levels by increasing cholesterol accumulation in BM progenitor cells. However, increased adipose tissue inflammation in DDC-fed mice (25) could be partly responsible for the increased monocyte levels, as we have recently demonstrated that adipose tissue inflammation can also result in monocytosis and increased levels of circulating Ly6 $\mathrm{C}^{\mathrm{hi}}$ monocytes (52).

We therefore propose, as illustrated schematically in Figure 9, that increased circulating levels of Ly6 $\mathrm{C}^{\text {hi }}$ monocytes and increased cell-surface levels of CCR2 on Ly6C $\mathrm{C}^{\text {hi }}$ monocytes in DDC-fed mice exacerbate ath- 
erosclerosis progression in arterial areas with increased arterial glucose metabolism and resulting CCL2 secretion. Thus, a dysregulated feed-forward mechanism exists in which SMC glucose flux augments inflammatory-driven CCL2 expression in these cells, attracting additional monocytes to the growing lesion. A similar feed-forward mechanism has recently been proposed by Verzola et al. in which myostatin increases CCL2-dependent chemotaxis of monocytes and CCL2, in turn, augments myostatin expression in SMCs (53). However, it is possible that additional mechanisms contribute to the phenotype observed in the present study.

How does increased glucose uptake through GLUT1 enhance inflammatory activation of SMCs? Our results suggest that the ability of TNF- $\alpha$ to induce CCL2 secretion is severely blunted by inhibiting glycolysis and aldose reductase activity and that glycolysis, as well as the polyol pathway, are enhanced by GLUT1 overexpression. This is consistent with recent studies in macrophages stimulated with LPS (13). However, we did not find significantly elevated levels of oxidation markers or succinate in GLUT1-overexpressing SMCs, as has been shown to mediate the inflammatory effects in macrophages activated by LPS (54), nor were we able to alter the inflammatory effects of TNF- $\alpha$ by altering succinate levels. More likely, mediators of the GLUT1-induced SMC inflammatory phenotype are sorbitol and/or glyceraldehyde, or other intermediates in the polyol pathway, perhaps generated through increased glycation of intracellular proteins or by oxidative processes not detected in our metabolomics screen.

In the present study, overexpression of GLUT1 in myeloid cells did not promote atherosclerosis in $L d l r^{--}$DDC-fed mice, consistent with our previous studies in low-fat-fed lean $\mathrm{Ldlr}^{-1}$ mice (14). Thus, increased SMC expression of GLUT1 appears to provide a stronger proatherogenic effect than do myeloid cell GLUT1 levels. Interestingly, knockdown of GLUT1 in hematopoietic cells has been shown to prevent myelopoiesis and atherosclerosis in Apoe ${ }^{-/-}$mice (55). The effect of GLUT1 knockdown in SMCs is so far unknown but would be predicted to increase SMC apoptosis (19).

Together, this study demonstrates a potentially novel mechanism whereby arterial glucose metabolism strikingly enhances atherosclerosis and formation of advanced lesions in a mouse model of metabolic syndrome. Although the human relevance of our findings remains to be investigated, our study suggests that aggressive treatment of dyslipidemia and other CVD risk factors may be particularly important in patients with metabolic syndrome in order to prevent the synergistic effects of arterial glucose metabolism on lesion progression.

\section{Methods}

Animals. Male $\mathrm{Ldlr^{-/ }}$ mice on the C57BL/6 background were fed a DDC (F4997; BioServ), which contains $60 \%$ calories from fat $(25,26)$ for 22 weeks to allow advanced atherosclerotic lesions to develop in the BCA. These mice were used to assess GLUT1 (Slc2a1) mRNA in BCA cross-sections by in situ hybridization. To investigate the effect of GLUT1 overexpression in SMCs, experimental animals were generated by crossing transgenic mice heterozygous for the human SLC2A1 transgene under control of the SM22 $\alpha$ promoter (24) with $L d l^{-1-}$ mice (both on the C57BL/6 background; the Jackson Laboratory). The resulting transgenic pups were backcrossed into the $L d l r^{--}$colony, producing transgenic mice that were also deficient in the Ldlrgene, allowing for a lipoprotein profile in which atherosclerosis can develop $(34,56)$. Mice were maintained by crossing male $L d l r^{--}$mice containing the SLC2A1 transgene to $L d l^{\prime-}$ females. For all studies, $L d r^{\prime-}$ mice that overexpress $S L C 2 A 1$ in 1 allele were used as experimental mice (SM-GLUT1) and their $L d l r^{-/}$littermates without the $S L C 2 A 1$ transgene served as controls (WT). Because littermates were used, the numbers of mice in each group are not identical.

To investigate the role of GLUT1 overexpression in macrophages, GLUT1 was overexpressed in myeloid cells under control of the CD68 promoter, as previously described (14). Briefly, BM stem cells were transduced with a CD68-GLUT1 or CD68-EGFP retrovirus as a control and transplanted into lethally irradiated (10 Gy) C57BL/ 6 recipients ( $5 \times 10^{6}$ cells injected retro-orbitally). After being allowed 7 weeks to recover, BM was harvested again and transplanted into irradiated 8- to 10 -week-old $\mathrm{Ldll}^{\prime-}$ mice. Male and female mice, 8-12 weeks of age, were used for experiments.

For atherosclerosis studies, mice were fed regular chow, a low-fat semi-purified diet described previously (34), or DDC, known to produce a phenotype characteristic of metabolic syndrome (25). Body weight and blood glucose were monitored every 4 weeks. A glucose tolerance test (GTT) was done after 13 weeks of DDC feeding by i.p. injection of dextrose ( $1 \mathrm{~g} / \mathrm{kg}$, Baxter) in fasted (4-6 hours) mice. Blood glucose was monitored throughout the study and for the GTT using One Touch Ultra test strips (LifeScan). A mouse hemoglobin A1c kit from Crystal Chem (catalog 80310) was used to measure HbA1c levels. Nonfasting plasma lipids were determined in the morning by colorimetric assays according to manufacturers' instructions; lipids included triglycerides (MilliporeSigma), and cholesterol (Wako). 
Isolation and maintenance of aortic SMCs. Mouse aortic SMCs were isolated and cultured as previously described (57). In short, thoracic aortas were harvested from 3- to 4-week-old mice, cleaned of residual fatty tissue under a dissecting microscope, and incubated in DMEM supplemented with $5.5 \mathrm{mM}$ glucose, $1 \%$ fungizone, $1 \%$ penicillin/streptomycin, $2 \mathrm{mg} / \mathrm{ml} \mathrm{BSA}$ (MilliporeSigma, A-4378), $1 \mathrm{mg} / \mathrm{ml}$ collagenase type II (Worthington, CLS-2), $0.375 \mathrm{mg} / \mathrm{ml}$ soybean trypsin inhibitor (Worthington), and $0.2305 \mathrm{mg} / \mathrm{ml}$ elastase (Worthington, LS00290) for 3 minutes. After incubation, the aortas were put back into ice-cold DMEM supplemented with $1 \%$ fungizone and $1 \%$ penicillin/streptomycin. Aortas were then cut longitudinally, the adventitia peeled away, and the endothelial cells removed with a sterilized cotton swab. The aortic media was cut into 5-mm pieces; placed in DMEM supplemented with $1 \%$ fungizone, $1 \%$ penicillin/ streptomycin, $20 \% \mathrm{FBS}$, and $0.8 \mathrm{mg} / \mathrm{ml}$ collagenase type II; and incubated in a $37^{\circ} \mathrm{C}$ water bath for $\sim 20$ minutes. The supernatant was removed after centrifugation and the pellet was washed once with DMEM and then resuspended in DMEM supplemented with 1\% fungizone, 1\% penicillin/streptomycin, 20\% FBS, $0.8 \mathrm{mg} / \mathrm{ml}$ collagenase type II, and $0.44 \mathrm{mg} / \mathrm{ml}$ elastase; it was then incubated in a $37^{\circ} \mathrm{C}$ water bath for 45-50 minutes with periodic mixing. The digested cells were resuspended in DMEM supplemented with $1 \%$ fungizone, $1 \%$ penicillin/streptomycin, $1 \%$ nonessential amino acids (NEAA), and 20\% FBS and plated onto tissue culture plates. Cells were maintained in DMEM supplemented with $1 \%$ fungizone, $1 \%$ penicillin/streptomycin, 1\% NEAA, 20\% FBS (10\% FBS after passage 3), and $25 \mathrm{mM}$ glucose. The cells were used for experiments at passages 0-9. Cells were free of mycoplasma contamination, as determined by the MycoProbe Mycoplasma Detection Kit (R\&D Systems).

In some experiments, SMCs were treated in the presence or absence of TNF- $\alpha$ in the presence of a cell-permeable diethyl succinate (MilliporeSigma), or endogenous succinate levels were increased by incubation with diethyl butyl malonate (MilliporeSigma), an inhibitor of the mitochondrial succinate transporter, according to methods described by Mills et al. (54). In other experiments, glycolysis was inhibited by incubation of the SMCs in the presence of $1 \mathrm{mM}$ 2-DOG, as described by Tannahill et al. (13), or aldose reductase was inhibited by $30-100 \mu \mathrm{M}$ zopolrestat or $10-30 \mu \mathrm{M}$ tolrestat (both from MilliporeSigma and dissolved in DMSO). DMSO was added as vehicle to control cells.

Overexpression of GLUT1 in mouse SMCs. GLUT1 was overexpressed using an ecotropic pBM retroviral vector described previously (14). Isolated mouse aortic SMCs were serum starved and then incubated for 24 hours in the presence of viral media (diluted 1:30 in standard growth media), 1M HEPES, and 5.5 $\mu \mathrm{g} / \mathrm{ml}$ polybrene. Cells transduced by the empty $\mathrm{pBM}$ virus were used as controls. Transduced cells were selected in the presence of puromycin.

Isolation and maintenance of BMDMs. BMDMs were harvested from freshly obtained bones by syringe flushing with DMEM medium supplemented with $1 \%$ fungizone and $1 \%$ penicillin/streptomycin. Cells were purified of erythrocytes and maintained in DMEM media ( $25 \mathrm{mM}$ glucose) containing $1 \%$ fungizone, $1 \%$ penicillin/streptomycin, $7 \% \mathrm{FBS}$, and $30 \% \mathrm{~L}$ cell-conditioned media. Cells were allowed to differentiate for 7-10 days prior to being used in experiments.

Lesion analysis, in situ hybridization, histochemistry, and aortic lipid measurements. At the end of the atherosclerosis studies, tissues (except the aorta) were collected for histology in 4\% PFA, following perfusion with PBS. Aortas were immediately flushed with RNAlater (Invitrogen) using a 1-ml syringe and then dissected from the heart down to the femoral bifurcation and stored in RNAlater at $4^{\circ} \mathrm{C}$. The aortic sinus and BCA from each animal were paraffin-embedded and later serial sectioned for histological analysis by Movat's pentachrome stain, as described previously (58). Cross-sections were also analyzed by TUNEL staining to assess apoptosis, for expression of specific tissue markers including Ki-67 for proliferating cells, smooth muscle $\alpha$-actin, and Mac-2 as a macrophage marker. Hyaluronan was measured in BCA sections by using a biotinylated hyaluronan-binding protein (59). Adjacent BCA cross-sections were pretreated with hyaluronan lyase for 1 hour at $37^{\circ} \mathrm{C}$ as a control. Slc2al mRNA was assessed by in situ hybridization using an RNAscope assay according to manufacturer's instructions (Advanced Cell Diagnostics).

Within 1 month following the harvest, aortas were opened longitudinally and measured for en face lesion area while in RNAlater by an observer blinded to the treatment groups. Only thickened deposits on the endothelial side were counted positive as lesion area. Following lesion quantification, lipids were extracted from the aortas using a 3:2 mixture of hexane/isopropanol 2 times for 13 minutes at $4^{\circ} \mathrm{C}$. Extracted lipids were dried at $37^{\circ} \mathrm{C}$ under nitrogen and resuspended in PBS with sodium cholate and triton-X (Sigma Aldrich). Immediately after lipid extraction, aortas were homogenized (Tissue Tearor model 
985370-393 variable speed [Biospec products Inc.]) and digested with protease K (Qiagen), and mRNA was isolated from each aorta (Qiagen RNAeasy kit). Extracted lipids were later analyzed for cholesterol content using Amplex Red Cholesterol Assay Kit (Invitrogen) and for triglyceride content (MilliporeSigma).

Tracing of Ly6Chi monocytes into lesions of atherosclerosis. Ldlr ${ }^{-1}$ mice overexpressing GLUT1 in SMCs and WT $L d l r^{-1}$ littermate controls were fed DDC for 16 weeks to establish atherosclerotic lesions (Figure 8). Monocytes were depleted by retro-orbitally injecting clodronate liposomes (Encapsula NanoSciences; std macrophage depletion kit (catalog NC1253361); Thermo Fisher Scientific; $200 \mu 1 /$ mouse at $5 \mathrm{mg} / \mathrm{ml}$ of clodronate), as described previously (60). After verification (16 hours after clodronate injection) of monocyte depletion, yellow-green (YG) microsphere beads (Fluoresbrite YG Microspheres $0.5 \mu \mathrm{m}$; Thermo Fisher Scientific) were injected to selectively label Ly6 $\mathrm{C}^{\text {hi }}$ monocytes (60). Labeling efficiency of blood monocytes was determined by flow cytometry 3 days after YG-bead injection. Recruitment of bead-positive cells to the BCA was analyzed 4 days after bead injection. Briefly, BCAs were dissected, fixed, embedded in paraffin, and serial sectioned, and the number of beads were counted over the entire length of the BCA and averaged. The presence of lesions was determined using a Movat's pentachrome stain, and sections adjacent to sites of identified lesions were stained with an anti-Mac-2 antibody (Cedarlane, CL8942AP), mounted with DAPI-containing media, and visualized. YG-particles were counted by an investigator blinded to the treatment groups.

Glucose metabolism and metabolomics. Mouse aortic SMCs were isolated and plated in $25 \mathrm{mM}$ glucose DMEM supplemented with $1 \%$ penicillin/streptomycin, 1\% NEAA, and 20\% FBS. Glucose uptake was measured as uptake of $1 \mu \mathrm{Ci} / \mathrm{ml}$ of 2-DOG (25 Ci/mmol of 2-DOG; PerkinElmer) over a 24-48 hour period. Lactate release into the media was measured by a colorimetric assay (MilliporeSigma) over a 48-hour period. Targeted aqueous metabolomics was performed on isolated mouse aortic SMCs transduced with the empty pBM retroviral vector or GLUT1 vector through LC-MS/MS, using an Agilent 1260/AB-Sciex 5500 Qtrap LC-MS/MS instrument and previously described protocols (61). This system provides detailed information on metabolites involved in glycolysis, the TCA cycle, and pentose phosphate shunt, as well as amino acid, fatty acid, and nucleic acid metabolism. Stable isotope-labeled 2- $\mathrm{C}^{13}$-glutamic acid (150.0/85.0), 2-C $\mathrm{C}^{13}$-tyrosine (184.1/138.0), 3-C $C^{13}$-lactate (92.0/45.0), and $C^{13}$-glucose $(185.0 / 92.0)$ with a purity of $>99 \%$ were used as standards (Cambridge Isotope Labs). Typical median CV values were $7 \%-8 \%$ in a single 20 -minute run.

Measurements of hyaluronan secretion. The amount of secreted hyaluronan in SMC conditioned media was determined by a modified competitive ELISA in which the samples to be assayed were first mixed with biotinylated hyaluronan-binding protein and then added to hyaluronan-coated microtiter plates, as described by Mapleson and Buchwald (62).

Real-time PCR. Gene expression was quantified by real-time PCR. RNA isolation and the real-time PCR protocol were performed as described previously (63). Briefly, RNA was isolated using Qiagen RNeasy or Macherey-Nagel Nucleospin RNA kits according to manufacturer's protocol and treated with DNase1 ( $1 \mu \mathrm{g} /$ sample, Thermo Fisher Scientific) to remove trace DNA. Real-time PCR was performed using the SYBR Green 1 detection method (Thermo Fisher Scientific). Ct values were normalized to $R n 18 s$ and presented as fold over control. PCR-ready first-strand cDNA from human coronary artery from a 68-year old male was obtained from AMSBIO. See Supplemental Table 3 for list of primers used.

Flow cytometry. Retro-orbital blood was harvested and purified of erythrocytes. Fluorescently labeled antibodies were added after addition of viability dye (diluted 1:2; eBioscience, catalog 65-0863) and an Fc blocking step (eBioscience, catalog 14-0161). The antibodies used included APC-labeled anti-CD115 (undiluted; eBioscience, clone AFS98), PECy7-labeled anti-GR1 (diluted 1:20; eBioscience, clone RB68C5), FITC-labeled anti-CD45 (diluted 1:10; eBioscience, clone 30-F11), and PE-labeled anti-CCR2 (diluted 1:2; BioLegend, clone SA203G11). Cells were kept at $4^{\circ} \mathrm{C}$ during all steps in the staining protocol. Cells were assessed on a Canto RUO flow cytometer or, in some experiments, sorted on a FACS Aria 2 cell sorter directly into lysis buffer (RNeasy Micro Kit, Qiagen). Cells were gated by forward and side scatter to remove debris, doublets were removed by forward scatter-height vs. forward scatter-width, and cells were then gated on viability dye to remove dead cells. Ly6 $\mathrm{C}^{\text {hi }}$ monocytes were considered CD $45^{+} \mathrm{C}$ $\mathrm{D} 115^{+} \mathrm{GR} 1^{\text {hi }}$, Ly6 $6 \mathrm{C}^{\text {lo }}$ monocytes were $\mathrm{CD} 45^{+} \mathrm{CD} 115^{+} \mathrm{GR} 1^{\text {lo }}$, and neutrophils were $\mathrm{CD} 45^{+} \mathrm{CD} 115^{-} \mathrm{GR} 1^{\mathrm{hi}}$. All flow cytometry included appropriate fluorescence minus one (FMO) controls. Total cell counts were made using Cellometer Auto 2000 Cell Viability Counter (Nexcelom Bioscience). In sorted cells, mRNA from monocytes and neutrophils was extracted (RNeasy Micro Kit, Qiagen), and cDNA was made (SuperScript VILO, Thermo Fisher Scientific) according to manufacturer's instructions. Quantitative PCR for gene expression was performed as described above. 
Statistics. Statistics for all studies were performed using Prism GraphPad 7.0 software. Unpaired 2-tailed Student's $t$ test was used to compare 2 conditions, while multiple groups were compared by 1-way ANOVA with a Tukey's post hoc tests for normally distributed data. D'Agostino-Pearson omnibus normality tests were performed to evaluate if the data were normally distributed. Data not normally distributed were analyzed by Kruskal-Wallis tests followed by Dunn's multiple-comparison tests (multiple groups) or Mann-Whitney $U$ tests (2 groups). Statistical outliers were excluded based on Grubbs' tests, and all exclusions are reported in the figure legends. A $P$ value less than 0.05 was considered significant. For targeted metabolomics data sets, statistical analysis was performed by unpaired two-tailed $t$ tests corrected for multiple comparisons using the Holm-Sidak method. Error bars indicate \pm SEM, except for the results in Table 1, where \pm SDs are reported.

Study approval. All experiments were performed in accordance with an approved University of Washington IACUC (protocol 3154-01).

\section{Author contributions}

VZW, SB, FK, JEK, MSA, and NA performed the study. TNW and JLH provided reagents, provided advice on experimental design, and edited the manuscript. VZW and KEB conceived the study, analyzed the data, and wrote the manuscript.

\section{Acknowledgments}

This study was supported by NIH grants R01HL126028, R01HL062887, and P01HL092969 and a grant from the American Heart Association, 14GRNT20410033, to KEB. Part of the study was supported by the Diabetes Research Center at the University of Washington, P30DK017047. The metabolomics studies were performed by the Analytic Core of the University of Washington NORC supported by P30DK035816. VZW was supported by the Samuel and Althea Stroum Fellowship in Diabetes Research.

Address correspondence to: Karin Bornfeldt, University of Washington School of Medicine South Lake Union Campus, 850 Republican Street, Seattle, Washington 98109, USA. Phone: 206.543.1681; Email: bornf@uw.edu.

1. Silverman MG, et al. Association Between Lowering LDL-C and Cardiovascular Risk Reduction Among Different Therapeutic Interventions: A Systematic Review and Meta-analysis. JAMA. 2016;316(12):1289-1297.

2. [No authors listed]. Randomised trial of cholesterol lowering in 4444 patients with coronary heart disease: the Scandinavian Simvastatin Survival Study (4S). Lancet. 1994;344(8934):1383-1389.

3. Cannon $\mathrm{CP}$, et al. Intensive versus moderate lipid lowering with statins after acute coronary syndromes. $N$ Engl J Med. 2004;350(15):1495-1504.

4. Ray KK, et al. Early and late benefits of high-dose atorvastatin in patients with acute coronary syndromes: results from the PROVE IT-TIMI 22 trial. J Am Coll Cardiol. 2005;46(8):1405-1410.

5. Lee $\mathrm{KH}$, et al. Benefit of early statin therapy in patients with acute myocardial infarction who have extremely low low-density lipoprotein cholesterol. J Am Coll Cardiol. 2011;58(16):1664-1671.

6. Howson JMM, et al. Fifteen new risk loci for coronary artery disease highlight arterial-wall-specific mechanisms. Nat Genet. 2017;49(7):1113-1119.

7. Tacke F, et al. Monocyte subsets differentially employ CCR2, CCR5, and CX3CR1 to accumulate within atherosclerotic plaques. J Clin Invest. 2007;117(1):185-194.

8. Tabas I, Bornfeldt KE. Macrophage Phenotype and Function in Different Stages of Atherosclerosis. Circ Res. 2016;118(4):653-667.

9. Ross R. Atherosclerosis--an inflammatory disease. N Engl J Med. 1999;340(2):115-126.

10. Libby P. Inflammation in atherosclerosis. Arterioscler Thromb Vasc Biol. 2012;32(9):2045-2051.

11. Libby P, Ridker PM, Hansson GK. Progress and challenges in translating the biology of atherosclerosis. Nature. 2011;473(7347):317-325.

12. Vats D, et al. Oxidative metabolism and PGC-1beta attenuate macrophage-mediated inflammation. Cell Metab. 2006;4(1):13-24

13. Tannahill GM, et al. Succinate is an inflammatory signal that induces IL-1 $\beta$ through HIF-1 $\alpha$. Nature. 2013;496(7444):238-242.

14. Nishizawa T, et al. Testing the role of myeloid cell glucose flux in inflammation and atherosclerosis. Cell Rep. 2014;7(2):356-365.

15. Peiró C, et al. Inflammation, glucose, and vascular cell damage: the role of the pentose phosphate pathway. Cardiovasc Diabetol. 2016;15:82.

16. Tarkin JM, Joshi FR, Rudd JH. PET imaging of inflammation in atherosclerosis. Nat Rev Cardiol. 2014;11(8):443-457.

17. Figueroa AL, et al. Measurement of arterial activity on routine FDG PET/CT images improves prediction of risk of future CV events. JACC Cardiovasc Imaging. 2013;6(12):1250-1259.

18. Iwaki T, Mizuma H, Hokamura K, Onoe H, Umemura K. [18F]FDG Uptake in the Aortic Wall Smooth Muscle of Atherosclerotic Plaques in the Simian Atherosclerosis Model. Biomed Res Int. 2016;2016:8609274

19. Hall JL, Chatham JC, Eldar-Finkelman H, Gibbons GH. Upregulation of glucose metabolism during intimal lesion formation is coupled to the inhibition of vascular smooth muscle cell apoptosis. Role of GSK3beta. Diabetes. 2001;50(5):1171-1179.

20. Gomez D, Owens GK. Smooth muscle cell phenotypic switching in atherosclerosis. Cardiovasc Res. 2012;95(2):156-164. 
21. Bashan N, Burdett E, Hundal HS, Klip A. Regulation of glucose transport and GLUT1 glucose transporter expression by O2 in muscle cells in culture. Am J Physiol. 1992;262(3 Pt 1):C682-C690.

22. Loike JD, Cao L, Brett J, Ogawa S, Silverstein SC, Stern D. Hypoxia induces glucose transporter expression in endothelial cells. Am J Physiol. 1992;263(2 Pt 1):C326-C333.

23. Parathath $\mathrm{S}$, et al. Hypoxia is present in murine atherosclerotic plaques and has multiple adverse effects on macrophage lipid metabolism. Circ Res. 2011;109(10):1141-1152.

24. Adhikari N, et al. Increase in GLUT1 in smooth muscle alters vascular contractility and increases inflammation in response to vascular injury. Arterioscler Thromb Vasc Biol. 2011;31(1):86-94.

25. Subramanian S, et al. Dietary cholesterol worsens adipose tissue macrophage accumulation and atherosclerosis in obese LDL receptor-deficient mice. Arterioscler Thromb Vasc Biol. 2008;28(4):685-691.

26. Averill MM, et al. S100A9 differentially modifies phenotypic states of neutrophils, macrophages, and dendritic cells: implications for atherosclerosis and adipose tissue inflammation. Circulation. 2011;123(11):1216-1226.

27. Hall JL, Gibbons GH, Chatham JC. IGF-I promotes a shift in metabolic flux in vascular smooth muscle cells. Am J Physiol Endocrinol Metab. 2002;283(3):E465-E471.

28. Yu FX, Chai TF, He H, Hagen T, Luo Y. Thioredoxin-interacting protein (Txnip) gene expression: sensing oxidative phosphorylation status and glycolytic rate. J Biol Chem. 2010;285(33):25822-25830.

29. Schulze PC, Yoshioka J, Takahashi T, He Z, King GL, Lee RT. Hyperglycemia promotes oxidative stress through inhibition of thioredoxin function by thioredoxin-interacting protein. J Biol Chem. 2004;279(29):30369-30374.

30. Katz SS, Shipley GG, Small DM. Physical chemistry of the lipids of human atherosclerotic lesions. Demonstration of a lesion intermediate between fatty streaks and advanced plaques. J Clin Invest. 1976;58(1):200-211.

31. Small DM. George Lyman Duff memorial lecture. Progression and regression of atherosclerotic lesions. Insights from lipid physical biochemistry. Arteriosclerosis. 1988;8(2):103-129.

32. Pyla R, Poulose N, Jun JY, Segar L. Expression of conventional and novel glucose transporters, GLUT1, -9, -10, and -12, in vascular smooth muscle cells. Am J Physiol, Cell Physiol. 2013;304(6):C574-C589.

33. Wight TN. Arterial remodeling in vascular disease: a key role for hyaluronan and versican. Front Biosci. 2008;13:4933-4937.

34. Renard CB, et al. Diabetes and diabetes-associated lipid abnormalities have distinct effects on initiation and progression of atherosclerotic lesions. J Clin Invest. 2004;114(5):659-668.

35. Hultgårdh-Nilsson A, Lövdahl C, Blomgren K, Kallin B, Thyberg J. Expression of phenotype- and proliferation-related genes in rat aortic smooth muscle cells in primary culture. Cardiovasc Res. 1997;34(2):418-430

36. Chai S, et al. Overexpression of hyaluronan in the tunica media promotes the development of atherosclerosis. Circ Res. 2005;96(5):583-591

37. Evanko SP, Angello JC, Wight TN. Formation of hyaluronan- and versican-rich pericellular matrix is required for proliferation and migration of vascular smooth muscle cells. Arterioscler Thromb Vasc Biol. 1999;19(4):1004-1013.

38. Yang L, et al. Lactate Promotes Synthetic Phenotype in Vascular Smooth Muscle Cells. Circ Res. 2017;121(11):1251-1262

39. Wang D, et al. High glucose and interleukin $1 \beta$-induced apoptosis in human umbilical vein endothelial cells involves in down-regulation of monocarboxylate transporter 4. Biochem Biophys Res Commun. 2015;466(4):607-614.

40. Butcher MJ, Waseem TC, Galkina EV. Smooth Muscle Cell-Derived Interleukin-17C Plays an Atherogenic Role via the Recruitment of Proinflammatory Interleukin-17A+ T Cells to the Aorta. Arterioscler Thromb Vasc Biol. 2016;36(8):1496-1506.

41. Alexander MR, Murgai M, Moehle CW, Owens GK. Interleukin-1ß modulates smooth muscle cell phenotype to a distinct inflammatory state relative to PDGF-DD via NF-кB-dependent mechanisms. Physiol Genomics. 2012;44(7):417-429.

42. Manetsch M, et al. TLR2 ligand engagement upregulates airway smooth muscle TNF $\alpha$-induced cytokine production. Am $J$ Physiol Lung Cell Mol Physiol. 2012;302(9):L838-L845.

43. Odaka M, et al. Differential regulation of chemokine expression by Th1 and Th2 cytokines and mechanisms of eotaxin/CCL11 expression in human airway smooth muscle cells. Int Arch Allergy Immunol. 2007;143 Suppl 1:84-88.

44. Schultz K, Murthy V, Tatro JB, Beasley D. Endogenous interleukin-1 alpha promotes a proliferative and proinflammatory phenotype in human vascular smooth muscle cells. Am J Physiol Heart Circ Physiol. 2007;292(6):H2927-H2934.

45. Rapp JH, Connor WE, Lin DS, Inahara T, Porter JM. Lipids of human atherosclerotic plaques and xanthomas: clues to the mechanism of plaque progression. J Lipid Res. 1983;24(10):1329-1335.

46. Combadière C, et al. Combined inhibition of CCL2, CX3CR1, and CCR5 abrogates Ly6C(hi) and Ly6C(lo) monocytosis and almost abolishes atherosclerosis in hypercholesterolemic mice. Circulation. 2008;117(13):1649-1657.

47. Berg KE, et al. Elevated CD14++CD16- monocytes predict cardiovascular events. Circ Cardiovasc Genet. 2012;5(1):122-131.

48. Murphy AJ, Dragoljevic D, Tall AR. Cholesterol efflux pathways regulate myelopoiesis: a potential link to altered macrophage function in atherosclerosis. Front Immunol. 2014;5:490.

49. Swirski FK, et al. Ly-6Chi monocytes dominate hypercholesterolemia-associated monocytosis and give rise to macrophages in atheromata. J Clin Invest. 2007;117(1):195-205.

50. Averill LE, Meagher RC, Gerrity RG. Enhanced monocyte progenitor cell proliferation in bone marrow of hyperlipemic swine. Am J Pathol. 1989;135(2):369-377.

51. Feldman DL, Mogelesky TC, Liptak BF, Gerrity RG. Leukocytosis in rabbits with diet-induced atherosclerosis. Arterioscler Thromb. 1991;11(4):985-994.

52. Nagareddy PR, et al. Adipose tissue macrophages promote myelopoiesis and monocytosis in obesity. Cell Metab. 2014;19(5):821-835.

53. Verzola D, et al. Myostatin mediates abdominal aortic atherosclerosis progression by inducing vascular smooth muscle cell dysfunction and monocyte recruitment. Sci Rep. 2017;7:46362

54. Mills EL, et al. Succinate Dehydrogenase Supports Metabolic Repurposing of Mitochondria to Drive Inflammatory Macrophages. Cell. 2016;167(2):457-470.e13.

55. Sarrazy V, et al. Disruption of Glut1 in Hematopoietic Stem Cells Prevents Myelopoiesis and Enhanced Glucose Flux in Ather omatous Plaques of ApoE(-/-) Mice. Circ Res. 2016;118(7):1062-1077.

56. MacDougall ED, et al. Aggressive very low-density lipoprotein (VLDL) and LDL lowering by gene transfer of the VLDL 
receptor combined with a low-fat diet regimen induces regression and reduces macrophage content in advanced atherosclerotic lesions in LDL receptor-deficient mice. Am J Pathol. 2006;168(6):2064-2073.

57. Askari B, et al. Rosiglitazone inhibits acyl-CoA synthetase activity and fatty acid partitioning to diacylglycerol and triacylglycerol via a peroxisome proliferator-activated receptor-gamma-independent mechanism in human arterial smooth muscle cells and macrophages. Diabetes. 2007;56(4):1143-1152.

58. Johansson F, et al. Type 1 diabetes promotes disruption of advanced atherosclerotic lesions in LDL receptor-deficient mice. Proc Natl Acad Sci USA. 2008;105(6):2082-2087.

59. Evanko SP, Raines EW, Ross R, Gold LI, Wight TN. Proteoglycan distribution in lesions of atherosclerosis depends on lesion severity, structural characteristics, and the proximity of platelet-derived growth factor and transforming growth factor-beta. $\mathrm{Am}$ J Pathol. 1998;152(2):533-546.

60. Kanter JE, et al. A Novel Strategy to Prevent Advanced Atherosclerosis and Lower Blood Glucose in a Mouse Model of Metabolic Syndrome. Diabetes. 2018;67(5):946-959.

61. Zhu J, et al. Colorectal cancer detection using targeted serum metabolic profiling. J Proteome Res. 2014;13(9):4120-4130.

62. Mapleson JL, Buchwald M. Effect of cycloheximide and dexamethasone phosphate on hyaluronic acid synthesis and secretion in cultured human skin fibroblasts. J Cell Physiol. 1981;109(2):215-222.

63. Kanter JE, et al. Diabetes promotes an inflammatory macrophage phenotype and atherosclerosis through acyl-CoA synthetase 1. Proc Natl Acad Sci USA. 2012;109(12):E715-E724. 